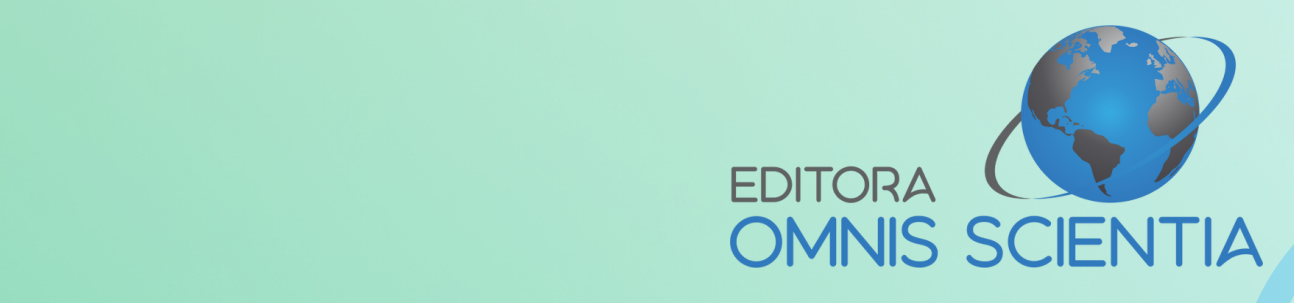

PESQUISA, SAÚDE E GRADUAÇÃO: MONOGRAFIAS QUE ENTRELAÇAM E CONTRIBUEM PARA O SER-PROFISSIONAL

Organizadores:

Prisca Dara Lunieres Pêgas Coêlho Francisco Railson Bispo de Barros

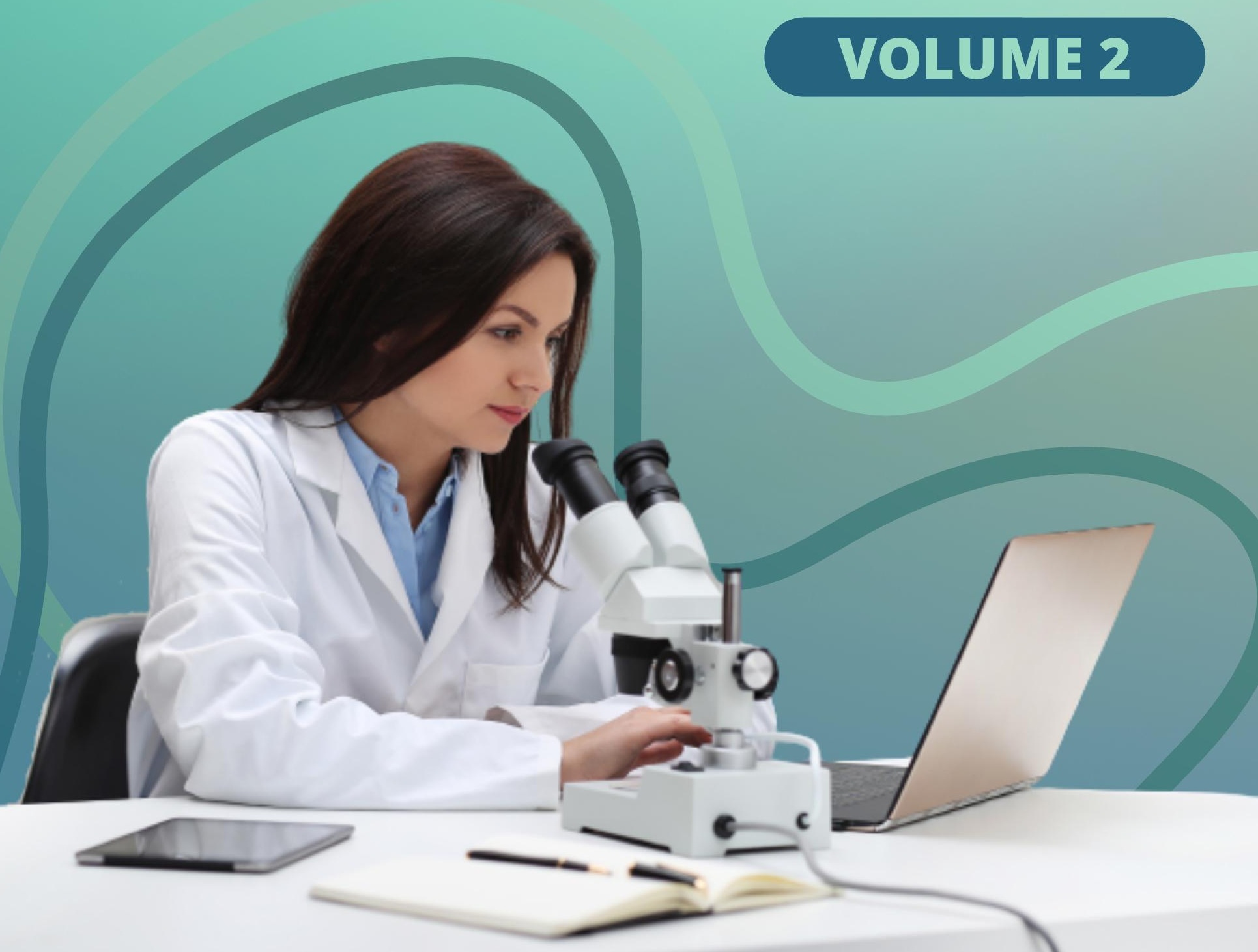




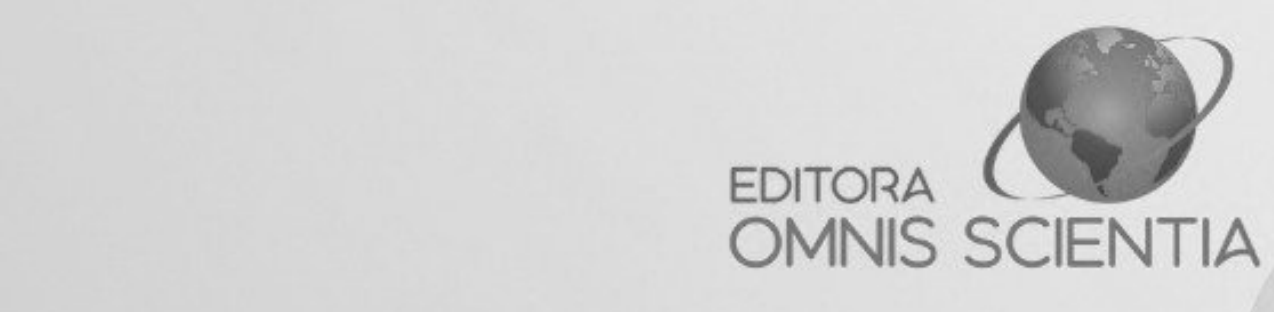

PESQUISA, SAÚDE E GRADUAÇÃO: MONOGRAFIAS QUE ENTRELAÇAM E CONTRIBUEM PARA O SER-PROFISSIONAL

Organizadores:

Prisca Dara Lunieres Pêgas Coêlho Francisco Railson Bispo de Barros

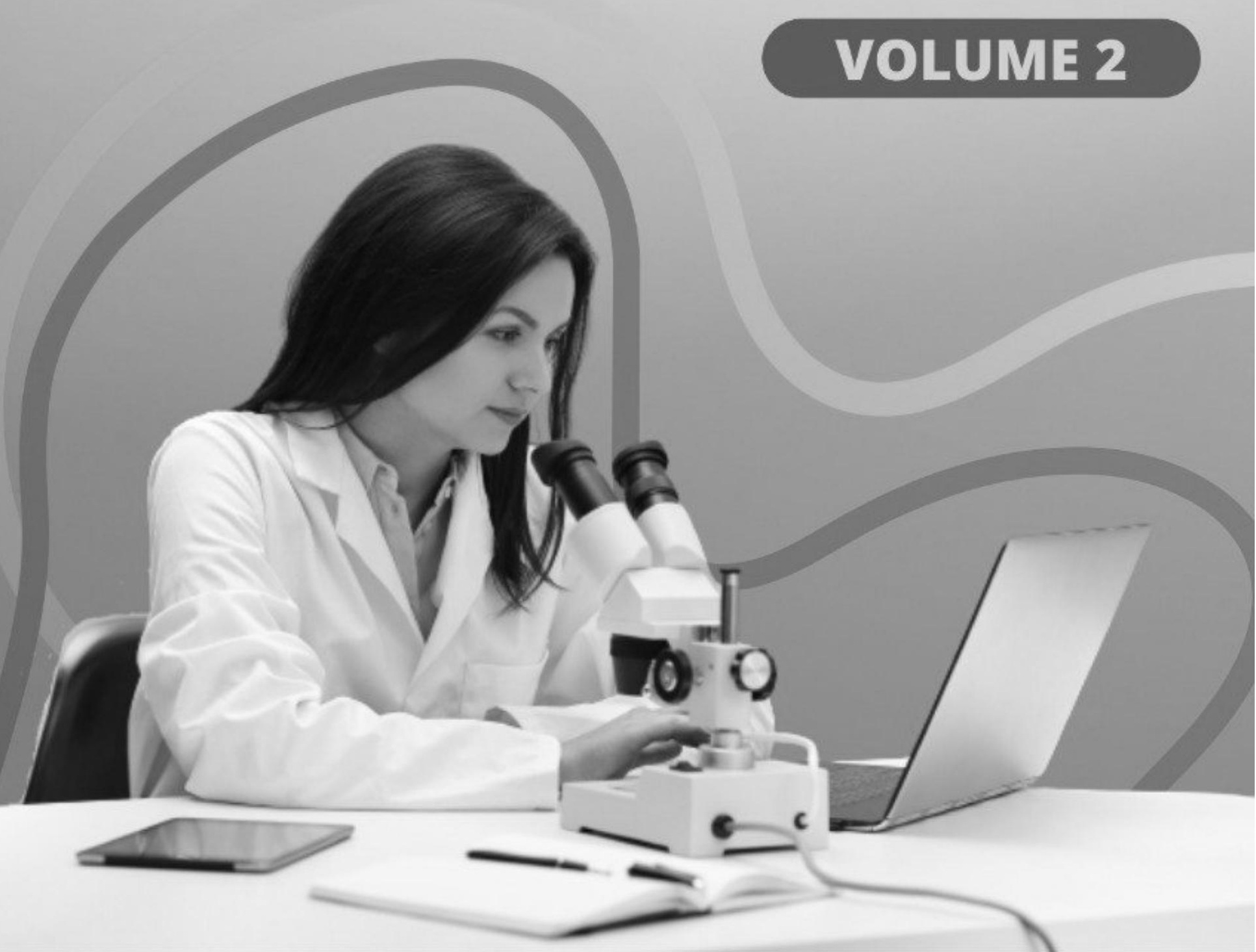


Editora Omnis Scientia

PESQUISA, SAÚDE E GRADUAÇÃO:

MONOGRAFIAS QUE ENTRELAÇAM E CONTRIBUEM PARA O SER-PROFISSIONAL

Volume 2

$1^{\text {a Edição }}$

TRIUNFO - PE 


\section{Editor-Chefe}

Me. Daniel Luís Viana Cruz

\section{Organizadores}

Prisca Dara Lunieres Pêgas Coêlho

Francisco Railson Bispo de Barros

\section{Conselho Editorial}

Dr. Cássio Brancaleone

Dr. Marcelo Luiz Bezerra da Silva

Dra. Pauliana Valéria Machado Galvão

Dr. Plínio Pereira Gomes Júnior

Dr. Walter Santos Evangelista Júnior

Dr. Wendel José Teles Pontes

Editores de Área - Ciências da Saúde

Dra. Camyla Rocha de Carvalho Guedine

Dra. Cristieli Sérgio de Menezes Oliveira

Dr. Leandro dos Santos

Dr. Hugo Barbosa do Nascimento

Dr. Marcio Luiz Lima Taga

Dra. Pauliana Valéria Machado Galvão

\section{Assistente Editorial}

Thialla Larangeira Amorim

\section{Imagem de Capa}

Freepik

\section{Edição de Arte}

Vileide Vitória Larangeira Amorim

\section{Revisão}

Os autores

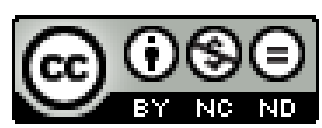

Este trabalho está licenciado com uma Licença Creative Commons - AtribuiçãoNãoComercial-SemDerivações 4.0 Internacional.

O conteúdo abordado nos artigos, seus dados em sua forma, correção e confiabilidade são de responsabilidade exclusiva dos autores. 


\section{Dados Internacionais de Catalogação na Publicação (CIP)}

(eDOC BRASIL, Belo Horizonte/MG)

P474 Pesquisa, saúde e graduação [livro eletrônico] : monografias que entrelaçam e contribuem para o ser-profissional: volume 2 / Organizadores Prisca Dara Lunieres Pêgas Coêlho, Francisco Railson Bispo de Barros. - Triunfo, PE: Omnis Scientia, 2022. 778 p. : il.

Formato: PDF

Requisitos de sistema: Adobe Acrobat Reader

Modo de acesso: World Wide Web

Inclui bibliografia

ISBN 978-65-88958-83-4

DOI 10.47094/978-65-88958-83-4

1. Enfermagem - Pesquisa - Brasil. 2. Ciências da saúde.

I.Coêlho, Prisca Dara Lunieres Pêgas. II. Barros, Francisco Railson

Bispo de.

CDD 610.73

Elaborado por Maurício Amormino Júnior - CRB6/2422

Editora Omnis Scientia

Triunfo - Pernambuco - Brasil

Telefone: +55 (87) 99656-3565

editoraomnisscientia.com.br

contato@editoraomnisscientia.com.br 


\section{PREFÁCIO}

Com prazer e satisfação compartilhamos o segundo volume desse e-book que reflete o resultado de pesquisas construídas por estudantes de graduação a partir da disciplina de Trabalho de Conclusão de Curso (TCC), que corresponde o semestre de 2021.2, do curso de Enfermagem, do Centro Universitário do Norte (UNINORTE/Ser Educacional) localizado em Manaus, capital do Amazonas. Sabemos que o TCC é um dos requisitos obrigatórios para a integralização curricular nos cursos de ensino superior. No entanto, viver e compartilhar a ciência é o que motiva a todos os envolvidos durante esse processo, desde coordenadores, professores e orientadores.

A proposta da disciplina de TCC é introduzir o estudante à pesquisa, incentivando-o a construir uma metodologia científica para detectar, conhecer e identificar fenômenos a partir de questionamentos e indagações identificados no quotidiano de ser e viver saudável e doente. E aqui, nessa teia de pesquisas, apresentamos a difícil arte de cuidar doentes e de autocuidado também, propondo ações que direcionem o ser-humano/ser-profissional em um caminho de cuidados em saúde pautados em uma prática baseada em evidências, sobretudo no contexto atual da saúde brasileira e mundial.

Por fim, como organizadores desse e-book, orgulhosamente saudamos a toda a equipe de discentes e docentes por tanto esforço e dedicação mesmo diante de uma realidade tão desafiadora pela pandemia do COVID-19, e ainda assim cumpriram com a responsabilidade em divulgar seus resultados como contribuição para a área da saúde e enfermagem frente aos mais diversos cenários e níveis de atenção. 


\section{SUMÁRIO}

CAPÍTULO 1. .28

EFEITOS DA PANDEMIA DE COVID-19 NA ENFERMAGEM COMO LINHA DE FRENTE

Admilson Tavares Assis Sobrinho

Amanda de Souza Fonseca

Aiury Oliveira de Freitas Serrão

Cleuciane da Silva Brito

Eduardo José do Nascimento Lima

Maciely Lopes Theodosio

Leandro Silva Pimentel

DOI: 10.47094/978-65-88958-83-4/28-39

CAPÍtULO 2. . .40

DESAFIOS NA IMPLANTAÇÃO DO PROCESSO DE ENFERMAGEM NO CONTEXTO DA ATENÇÃO PRIMÁRIA À SAÚDE: UMA REVISÃO INTEGRATIVA

Aluíno Tinoco da Silva

Janina Vences León

Sílvia Camacho da Silva

Sthefany Bezerra Borges de Araújo

Francisco Railson Bispo de Barros

Vilmar da Conceição Oliveira Filho

DOI: 10.47094/978-65-88958-83-4/40-50

CAPÍTULO 3 .51

A ATUAÇÃO DO ENFERMEIRO NA QUALIDADE DA COLETA DO EXAME PAPANICOLAU: UMA REVISÃO INTEGRATIVA

Andreza Oliveira de Lima

Angelina Maria Trindade dos Anjos

Elenilda Inácio Barreiros

Larissa da Silva Marques

\section{SUMÁRIO}


Mariluce Aires da Silva

Nabor Kina Júnior

Wivianne Lima Brito Góes

DOI: 10.47094/978-65-88958-83-4/51-61

CAPÍTULO 4 62

O USO DA ULTRASSONOGRAFIA NA PRÁTICA DIÁRIA DO ENFERMEIRO: UMA REVISÃO INTEGRATIVA

Antônio Luiz de Souza Félix

Francisco Anderson Lacerda de Araújo

Kalison Batista da Silva

Francisco Railson Bispo de Barros

Vilmar da Conceição Oliveira Filho

DOI: 10.47094/978-65-88958-83-4/62-73

CAPÍTULO 5 .74

A SAÚDE MENTAL DE ENFERMEIROS EM TEMPOS DE COVID-19

Beto Coelho Arcentales

Mirian Brasil Rodrigues

Francisco Railson Bispo de Barros

Vilmar da Conceição Oliveira Filho

DOI: 10.47094/978-65-88958-83-4/74-82

CAPÍTULO 6 83

IMPACTOS DO DIAGNÓSTICO DO TRANSTORNO DO ESPECTRO AUTISTA NA FAMÍLIA: CUIDADOS E CONTEXTO FAMILIAR

Bianca Raquel Araújo Campos

Lorenna Fernandes da Silva Bento

Francisca Magda de Souza Pinto Silva Xavier

DOI: 10.47094/978-65-88958-83-4/83-94

\section{SUMÁRIO}


CAPÍTULO 7

O IMPACTO DAS FAKE NEWS FRENTE À PANDEMIA COVID-19: UMA REVISÃO INTEGRATIVA

Brenda Rufino de Sousa

Herson Thiago Nunes Pitillo

Francisco Railson Bispo de Barros

Vilmar da Conceição Oliveira Filho

DOI: 10.47094/978-65-88958-83-4/95-104

CAPÍTULO 8 105

A ATUAÇÃo do ENFERMEIRO NA ASSISTÊNCIA AO PACIENTE ACOMETIDO POR GONORREIA

Brenno Eric de Sousa Silva

Fernanda Luiza Trajano Knights

Gilsivan Araújo dos Santos

Leandro Silva Pimentel

DOI: $10.47094 / 978-65-88958-83-4 / 105-115$

CAPÍTULO 9. 116

O PAPEL DO ENFERMEIRO NA PREVENÇÃO DO ÓBITO MATERNO E NEONATAL POR CAUSAS EVITÁVEIS: UMA REVISÃO INTEGRATIVA

Celina Moreira de Souza

Evellyn Cristine Pedrosa de Melo

Jessica Huchoua Giroux

Raimunda Souza Freitas Machado

Rayza Iara Santos Pereira

Francisco Railson Bispo de Barros

Vilmar da Conceição Oliveira Filho

DOI: 10.47094/978-65-88958-83-4/116-129

\section{SUMÁRIO}


ESTRATÉGIAS UTILIZADAS PELO ENFERMEIRO PARA PROMOÇÃO A SAÚDE DA PESSOA IDOSA: UMA REVISÃO INTEGRATIVA

Fabíola Jhullye França da Silva

Jordana Viana Pinheiro

Francisco Railson Bispo de Barros

Vilmar da Conceição Oliveira Filho

DOI: 10.47094/978-65-88958-83-4/130-143

CAPÍTULO 11

SAÚDE E COMPORTAMENTO DE IDOSOS CONVIVENDO COM O HIV: UMA REVISÃO INTEGRATIVA

Diva Raimunda Silva de Melo

Helenilce Mendes Cabral

Herilane Pereira Gama

Karen Batista de Souza

Linda Caroline Coelho Silva

Luiz Henrique da Cruz de Macedo

Francisco Railson Bispo de Barros

Vilmar da Conceição Oliveira Filho

DOI: 10.47094/978-65-88958-83-4/144-149

CAPÍTULO 12 .155

ASSISTÊNCIA DO ENFERMEIRO AO PACIENTE COM SÍFILIS CONGÊNITA

Joyce Neves Batista

Karina Correa da Silva

Leandro Silva Pimentel

DOI: 10.47094/978-65-88958-83-4/155-161 
PERFIL EPIDEMIOLÓGICO DA MORTALIDADE MATERNA NO AMAZONAS NOS ANOS DE 2009 A 2019

Beatriz Barbosa Figueiredo

Biancca Daniela Silva De Lima

Fábio Crispim Queiroz

Joyce Kelly Da Silva De Jesus

Larissa Luana Oliveira Dos Santos

Francisco Railson Bispo De Barros

Vilmar da Conceição Oliveira Filho

DOI: 10.47094/978-65-88958-83-4/162-171

CAPÍTULO 14 172

ASSISTÊNCIA DA EQUIPE DE SAÚDE NO PERÍODO GRAVÍDICO-PUERPERAL: UMA REVISÃO INTEGRATIVA

Lionella De Araújo Rêge

Marta Fabricia Passos De Lima Oliveira

Raquel Panaifo De Araujo

Richard Meneses Da Cunha

Viviam Gama Azevedo

Francisco Railson Bispo De Barros

Vilmar da Conceição Oliveira Filho

DOI: 10.47094/978-65-88958-83-4/172-181

CAPÍTULO 15 182

ASSISTÊNCIA DE ENFERMAGEM NO PARTO HUMANIZADO: REVISÃO INTEGRATIVA DA LITERATURA

Camila Cristina Araújo Silva

Lillian de Souza Vasconcelos

Mélane Vasconcelos Oliveira

Mellyssa Souza da Silva

Safira da Silva marialva

\section{SUMÁRIO}


Thyssia Rodrigues Figueiredo Collins

Wivianne Lima Brito Góes

DOI: 10.47094/978-65-88958-83-4/182-193

CAPÍTULO 16 .194

O IMPACTO DA DIABETES MELLITUS TIPO II NA QUALIDADE DE VIDA DE SEUS PORTADORES

Elias Matute Gomes

Jane Delfino da Silva

Pollyanne Buzaglo Rodrigues

Renan Guimarães de Azevedo

Rhuany Caroline Pimentel Bessa

Tatiane de Nazaré Souza Marques

Renata Schmitt

DOI: 10.47094/978-65-88958-83-4/194-205

CAPÍTULO 17. .206

PRINCIPAIS COMPLICAÇÕES DO PARTO CESÁREO: UMA REVISÃO DE LITERATURA

Renner Lopes Hermes

Thiffany Camacho Cespedes

Francisca Magda de Souza Pinto Silva Xavier

DOI: $10.47094 / 978-65-88958-83-4 / 206-216$

CAPÍTULO 18 .217

FATORES QUE INTERFEREM NA AMAMENTAÇÃO EXCLUSIVA ATÉ OS 6 MESES DE VIDA: REVISÃO INTEGRATIVA

Juliana de Oliveira Albuquerque

Nohanna Barbosa Lima

Valerie Rhaysa Liborio Silva de Oliveira

Francisca Magda de Sousa Pinto Silva Xavier

DOI: 10.47094/978-65-88958-83-4/217-226

SUMÁRIO 
A INFLUENCIA DA QUALIDADE DO CONHECIMENTO FRENTE AO CUIDADO DO IDOSO NA ASSISTÊNCIA DE ENFERMAGEM

Jailton Carlos Almeida de Matos

Jennifer Karla da Costa Andrade

Marcela Soares da Silva

Victória Violeta Fernandes Menescal

Wivianne Lima Brito Góes

DOI: 10.47094/978-65-88958-83-4/227-236

\section{CAPÍTULO 20}

PAPEL DO ENFERMEIRO FRENTE AOS CUIDADOS À PACIENTES TERMINAIS

Adiele Freitas Bertino

Alessandra Gomes Marques

Amanda Cavalcante Leal

Amanda Cavalcante de Souza

Karen Barbosa da Silva

Rallyson Ortigas dos Santos

Kadmiel Candido Chagas

DOI: 10.47094/978-65-88958-83-4/237-244

CAPÍTULO 21

O PAPEL DO ENFERMEIRO COM CUIDADOS PALIATIVOS AOS PACIENTES DE NEOPLASIA MAMARIA: REVISÃO INTEGRATIVA

Adriana Guimarães Araújo

Dyanne Priscilla Barros dos Santos

Marciele de Assis Coelho

Kadimiel Cândido Chagas

DOI: $10.47094 / 978-65-88958-83-4 / 245-255$

\section{SUMÁRIO}


A SEXUALIDADE NO ENVELHECIMENTO: O PAPEL DO ENFERMEIRO FRENTE AO IDOSO SOROPOSITIVO

Alexsander Nogueira da Silva

Amanda da Silva Lopes

Karoline Bulcão de Oliveira

Lucas Romário Macena Maia

Kadmiel Candido Chagas

DOI: 10.47094/978-65-88958-83-4/256-268

CAPÍTULO 23

ENFERMAGEM DERMATOLÓGICA: ATRIBUIÇÕES E CUIDADOS DO ENFERMEIRO NO TRATAMENTO DE PACIENTES COM CÂNCER DE PELE

Alice da Silva Lima

Jhessica dos Santos Gomes

Priscila Silva Aguiar

Kadmiel Cândido Chagas

DOI: 10.47094/978-65-88958-83-4/269-281

CAPÍTULO 24 .282

APLICATIVOS DE RELACIONAMENTOS GEOSSOCIAL COMO FATOR DE RISCO NA TRANSMISSÃO DE INFECÇÕES SEXUALMENTE TRANSMISSÍVEIS (IST’s)

Aline Swany Trindade de Aquino

Fellipe Barreto de Araújo

Ires Kethury Fernandes Eloi

Marta Trindade da Silva Sá

Taynná de Almeida Maduro

Kadmiel Cândido Chagas

DOI: 10.47094/978-65-88958-83-4/282-293

\section{SUMÁRIO}


CAPÍTULO 25

FAKE NEWS SOBRE VACINAS COMO POTENCIALIZADORAS DE PANDEMIAS

Amanda de Sousa Ferreira

Amanda Thalita de Paula Pinto

Ane Isabelly Fonseca Cintra

Maiara Oliveira da Cruz

Rayssa Brandão da Rocha

Neuliane Melo Sombra

Fabiane Veloso Soares

DOI: 10.47094/978-65-88958-83-4/294-305

CAPÍTULO 26 .306

AATUAÇÃO DO ENFERMEIRO NAASSISTÊNCIA DO PARTO HUMANIZADO: REVISÃO BIBLIOGRÁFICA

Ananda Gonçalves Curintima

Andréia Santana Silva

Elídia Mariscal Rubem

Joaquim Lucas Esteves de Almeida

Ketllen Sabrina da Silva

Rodrigo dos Santos Almeida

Kadmiel Candido Chagas

DOI: 10.47094/978-65-88958-83-4/306-316

CAPÍTULO 27.

A IMPORTÂNCIA DA PRESENÇA PATERNA NO PRÉ-NATAL

Ariel da Silva Maranhão

Ariane Da Silva Maranhão

Bruno Silva Palhão

Leonardo Rangel Ferreira Soares

Luciana Lima Moreno

Leandro Silva Pimentel 
CAPÍTULO 28

EVOLUÇÕES NO CAMPO DA ENFERMAGEM: HABILIDADES E COMPETÊNCIAS DO ENFERMEIRO NA ÁREA DERMATOLÓGICA E ESTÉTICA

Adriano Pacífico Rodrigues

Bruna Souza Brito

Carla Cristina Gomes da Costa

Kadmiel Cândido Chagas

DOI: 10.47094/978-65-88958-83-4/330-338

CAPÍTULO 29 339

TECNOLOGIA EDUCATIVA EM SAÚDE: DESMISTIFICANDO O SUPORTE BÁSICO DE VIDA PARA PROFISSIONAIS DA EDUCAÇÃO INFANTIL

Bruna de Souza Batista

Caroline Dias de Aguiar

Jardson Oliveira Batista

Jéssika Brasil Valério

Maria Thayná Maia dos Santos

Kadmiel Cândido Chagas

Prisca Dara Lunieres Pêgas Coêlho

DOI: 10.47094/978-65-88958-83-4/339-352

CAPÍTULO 30 353

FATORES DE RISCO À SAÚDE DE ADOLESCENTES ESCOLARES: ESTUDO DE REVISÃO DA LITERATURA

Cindy Zayda Batista Shapiama

Ingrid Beatriz Coelho de Souza

Joelma Batista da Silva

Vanessa Lima de Matos

Yago Bernardes de Araújo

Neuliane Melo Sombra

\section{SUMÁRIO}


CAPÍTULO 31 .368

SAÚdE PÚBLICA BRASILEIRA NO ENFRENTAMENTO DA PANDEMIA DO COVID 19

Claúdia dos Santos Tavares

Jardilene Marques Vieira

Jefferson Medeiros Castro

Roberta Yone dos Santos Rodrigues

Solane Mendonça da Costa

Ysa Carla Azevedo de Assis

Leandro Pimentel

DOI: 10.47094/978-65-88958-83-4/368-380

CAPÍTULO 32.

PRESENÇA DA DOULA NO PARTO NATURAL: UMA REVISÃO INTEGRATIVA SOBRE SEUS IMPACTOS E INFLUÊNCIAS

Daiana Gabriel Pereira

Giovana Alves Magalhães

Harthur Kayllon Gomes de Souza

Prisca Dara Lunieres Pegas Coelho

DOI: $10.47094 / 978-65-88958-83-4 / 381-393$

CAPÍTULO 33 .394

FAKE NEWS SOBRE SAÚDE NAS REDES MIDIÁTICAS E SUAS CONSEQUÊNCIAS Ariane Oliveira da Silva

Barbara Silva Gomes

Dayanna Oliveira da Costa

Natasha Lima da Silva

Tatiane de Souza Balieiro

Neuliane Melo Sombra

DOI: 10.47094/978-65-88958-83-4/394-406

\section{SUMÁRIO}


CAPÍTULO 34

O ESTRESSE COMO FATOR IMUNOSSUPRESSOR EM PROFISSIONAIS DE ENFERMAGEM

Débora Martins da Silva

Jamilly Mohana da Silva Alves

Laura Engels da Silva

Raquel Cordeiro Aleixo

Kadmiel Cândido

DOI: 10.47094/978-65-88958-83-4/407-421

CAPÍTULO 35

O IMPACTO DO PROCEDIMENTO DE EPISIOTOMIA NA VIDA DA MULHER

Brenda Cristina Reis De Souza

Flavia Brenda Pinho Dias

Lia De Souza Barros

Nayara Da Silva Gomes

Prisca Dara Lunieres Pegas Coelho

DOI: 10.47094/978-65-88958-83-4/422-440

CAPÍTULO 36

VACINAR A POPULAÇÃO: UMA ESTRATÉGIA PARA MANTER A SAÚdE PÚBLICA

SEM AMEAÇAS

Andreia Santos Lima

Fabiano Santos Pinho

Francisco Bruno Silva Cardozo

Glécio Gregório da Silva Gomes

Jéssica Karoline Rabelo Fialho

Márcia Souza da Fonseca

Michelle Souza Tribuzy

Leandro Silva Pimentel

DOI: 10.47094/978-65-88958-83-4/441-453

\section{SUMÁRIO}


CAPÍTULO 37

ASSISTÊNCIA DE ENFERMAGEM AS GESTANTES DIANTE DA PANDEMIA DA COVID-19

Amanda de Melo Farias

Erika Regina Nunes dos Santos

Geovanne Lima dos Santos

Maycon Henrique Garcia Fonseca

Oliver Khristian Caldas do Nascimento

Stefanie Lima Brandão

Thayanne Rafaela Mota Bandeira

Zegilson Ferreira Delmiro

Kadmiel Cândido Chagas

DOI: 10.47094/978-65-88958-83-4/454-462

CAPÍTULO 38 .463

ASSISTÊNCIA DE ENFERMAGEM NA PREVENÇÃO DA VIOLÊNCIA OBSTÉTRICA: REVISÃO INTEGRATIVA DA LITERATURA

Antônia Thalissa Farias Gomes

Fabiana Brito Abrahão Affonso

Glaucianne Holanda Batista

Víctor Lopes Barbosa

Viviane Hipi Gonçalves

Prisca Dara Lunieres Pêgas Coêlho

DOI: 10.47094/978-65-88958-83-4/463-479

CAPÍTULO 39 .480

\section{RELEVÂNCIA DO PARTO HUMANIZADO: UMA REVISÃO INTEGRATIVA}

Ana Fábia da Silva Feliciano

Cristian Marques de Oliveira

Hanna Brenda Silva Soares

Ilana Cristina da Silva Duarte 
Kelison Pantoja Maciel

Mateus da Silva Melo

Rejane Lima da Silva

Kadmiel Cândido Chagas

Thiago Henrique Souza de Castro Alves

DOI: 10.47094/978-65-88958-83-4/480-493

CAPÍTULO 40. .494

A MUSICOTERAPIA COMO FONTE DE CUIDADOS EM SAÚDE: UMA REVISÃo INTEGRATIVA

Jennifer Patricia Caldas Ferreira

Samuel Franklin Lelis Da Silva

Paula Marinho Borges ${ }^{3}$

Prisca Dara Lunieres Pêgas Coêlho

DOI: 10.47094/978-65-88958-83-4/494-509

CAPÍTULO 41 .510

INTERVENÇÃO DO ENFERMEIRO FRENTE AO PRÉ-NATAL TARDIO

Jessica da Redenção Fernandes

Prisca Dara Lunieres Pegas Coelho

DOI: 10.47094/978-65-88958-83-4/510-518

CAPÍTULO 42 .519

DESAFIOS DO ENFERMEIRO INTENSIVISTA NA ASSISTÊNCIA AO PACIENTE COM COVID-19: UMA REVISÃO INTEGRATIVA

Gilson Rogerio Becil de Oliveira

Jiovania Barbosa Maklouf de Oliveira

Kássia Cleandra Cruz Gomes

Priscila Ferreira Saraiva

Kadmiel Cândido Chagas

DOI: 10.47094/978-65-88958-83-4/519-531

SUMÁRIO 
HUMANIZAÇÃO DOS CUIDADOS DE ENFERMAGEM EM UNIDADE DE TERAPIA INTENSIVA: REVISAO INTEGRATIVA

Aldeiza Gonçalves de Oliveira

Amanda Rodrigues Freire

Crischinna de Souza da Conceição

Elyan Feitoza Palmeira

Karoline Lopes Ramiro

Prisca Dara Lunieres Pêgas Coêlho

DOI: 10.47094/978-65-88958-83-4/532-549

CAPÍTULO 44. .550

FATORES DE RISCO CARDIOVASCULAR MODIFICAVEIS EM INDÍGENAS COM HIPERTENSÃO ARTERIAL SISTÊMICA

Francisca Isa Souza Martins

Silvana Ketlen Magalhães Peres

Neuliane Melo Sombra

DOI: 10.47094/978-65-88958-83-4/550-561

CAPÍTULO 45 . .562

DESAFIOS ENFRENTADOS PELA ENFERMAGEM ANTES E DURANTE PANDEMIA DO COVID-19

Hortência Cardoso Vidal

Lady Mara Sena da Rocha

Larissa Vitória Soares da Silva

Prisca Dara Lunieres Pêgas Coêlho

Dayane Chimendes de Carvalho Lima

DOI: $10.47094 / 978-65-88958-83-4 / 562-572$ 
FATORES QUE INTERFEREM NA ADESÃO A PREVENÇÃO DO CÂNCER DE COLO DE ÚTERO

Adriana Nazário Silva

Ana Paula Muniz

Karina Carvalho dos Santos

Leila Karolaine de Oliveira dos Santos

Neiziane Freitas da Silva

Sandy Elen Marinho

Prisca Dara Lunieres Pêgas Coêlho

DOI: 10.47094/978-65-88958-83-4/573-583

CAPÍTULO 47 .584

ENFERMEIRO NO ACOLHIMENTO E CLASSIFICAÇÃO DE RISCO OBSTETRICA $(\mathrm{A} \& \mathrm{CR})$

Ialle Cristine da Silva

Lígia Lopes de Sousa

Kadmiel Cândido Chagas

DOI: 10.47094/978-65-88958-83-4/584-598

CAPÍTULO 48. .599

HUMANIZAÇÃO NA ENFERMAGEM EM TEMPO DE PANDEMIA E O IMPACTO PARA RECUPERAÇÃO DE PACIENTES

Fernanda Neves Teixeira

Graciele da Silva Carvalho

Luana Almeida Costa

Natássia Kíssia Barbosa do Nascimento

Orleane Aparecida Neves Jeffryes

Patrícia França de Freitas

Robson Bernardo Peres da Silva

Neuliane Melo Sombra

DOI: 10.47094/978-65-88958-83-4/599-611

\section{SUMÁRIO}


CONDUTAS DO ENFERMEIRO NA ESTRATÉGIA SAÚDE DA FAMÍLIA EM ATENÇÃO A SAÚDE DE MULHERES EM SITUAÇÃO DE VIOLÊNCIA

Estefane De Castro Carvalho

Keithy Damasceno Saraiva

Léia Reis de Souza

Madalena Aparecida De Lima

Tânia Maria de Sousa Coimbra

Neuliane Melo Sombra

DOI: 10.47094/978-65-88958-83-4/612-623

CAPÍTULO 50

INTERAÇÕES ENTRE A COVID-19 E O ACIDENTE VASCULAR CEREBRAL (AVC)

Alex Cardozo De Lima

Farezinho Guedes Ramires

Júlia Robert Miranda Geber

Julie Andrews Coelho De Souza

Kelves De Castro Alvarenga

Mailson Alves De Lima

Kadmiel Cândido Chagas

DOI: 10.47094/978-65-88958-83-4/624-633

CAPÍTULO 51

PRINCIPAIS DESAFIOS DOS ENFERMEIROS NO TRANSPORTE E RESGATE AEROMÉDICO DE PACIENTES COM COVID-19

Amanda Thais Francisco da Costa

Anaelle Monteiro dos Santos

Bárbara Quilim Soares

Bruna Cristina Campos da Silva

Meyri Hellen Viana da Silveira

Rosilane Amaral de Miranda 
Leandro Silva Pimentel

Adriano de Souza Gomes

DOI: 10.47094/978-65-88958-83-4/634-645

CAPÍTULO 52 .646

A SAÚDE MENTAL DOS PROFISSIONAIS DE ENFERMAGEM FRENTE À COVID-19: UMA REVISÃO DE BIBLIOGRÁFICA

Dhonleno Gomes dos Santos

Francineldo Ipuchima da Silva

Gabriel Ramalho dos Santos Moreno

João Paulo Simões Cabral

Milton Marques de Souza Júnior

Natanmara Ricardo da Silva

Valdilene de Souza Nogueira

Kadmiel Candido Chagas

DOI: 10.47094/978-65-88958-83-4/646-655

CAPÍTULO 53 .656

ASSISTENCIA DE ENFERMAGEM EM PACIENTES ADULTOS

OSTOMIZADOS: REVISÃO INTEGRATIVA DA LITERATURA

Jadma Silva de Almeida

José Nilton Pinheiro Do Carmo

Messias Carlos dos Santos

Ociney Souza dos Anjos

Thalya Menezes dos Santos

Kadmiel Cândido Chagas

DOI: 10.47094/978-65-88958-83-4/656-669

\section{SUMÁRIO}


ATUAÇÃO DOS ENFERMEIROS NOS CUIDADOS PALIATIVOS EM PACIENTES ONCOLÓGICOS

Ana Paula Almeida de Lima

Cléber Castro Paiva

Gabrielle Pereira Gomes Rosas

Leomara Oliveira dos Santos

Taís Karoline Barbosa

Kadmiel Candido Chagas

DOI: 10.47094/978-65-88958-83-4/670-680

CAPÍTULO 55. .681

ASSISTÊNCIA DE ENFERMGEM AO RECÉM-NASCIDO PREMATURO O PAPEL DO ENFERMEIRO NA UNIDADE DE TERAPIA INTENSIVA

Lucas de Araújo Lins

Mateus Gonçalves da Costa

Milena Brito de Oliveira

Rebeca Cardoso de Araújo Licor

Ronilson Cavalcante Matos

Prisca Dara Lunieres Pegas Coelho

DOI: 10.47094/978-65-88958-83-4/681-691

CAPÍTULO 56 .692

O PAPEL DO ENFERMEIRO NO COMBATE A VIOLÊNCIA OBSTÉTRICA

Aldeane de Lima Cordovil

Maria Raimunda Miranda Cardoso

Rebeka Karolyne Aleme Falcão

Regina Almeida de Silva e Silva

Prisca Dara Lunieres Pêgas Coelho

DOI: 10.47094/978-65-88958-83-4/692-703

\section{SUMÁRIO}


CUIDADOS DE ENFERMAGEM EM PACIENTES PORTADORES DE DIABETES MELLITUS: REVISÃO INTEGRATIVA

Auxiliadora Gonçalves Soares

Janeize Lopes Monteiro

Kerre Barbosa Lima

Rita Ariane Rodrigues Paes

Walderlane Tavares da Silva

Kadmiel Cândido Chagas

DOI: 10.47094/978-65-88958-83-4/704-714

CAPÍTULO 58. .715

ATRIBUIÇÕES DOS ENFERMEIROS FRENTE AOS DESAFIOS DA VACINAÇÃO DE COVID-19

Daniel Cristian Motta Maciel

Elenize da Silva de Souza

Inara de Amorim Ferreira

Sarah Renata da Silva Alves

Leandro Silva Pimentel

DOI: 10.47094/978-65-88958-83-4/715-725

CAPÍTULO 59 .726

DESAFIOS NA ASSISTÊNCIA DOS ENFERMEIROS NA INCLUSÃO DE PACIENTES SURDOS

Rosilene Moraes Leite

Sarah Christina de Souza Costa

Shirleny Shelry Ferreira Meireles

Tífani da Silva Monteiro

Neuliane Melo Sombra

DOI: 10.47094/978-65-88958-83-4/726-741

\section{SUMÁRIO}


CAPÍTULO 60

O USO DA OZONIOTERAPIA NO TRATAMENTO DE LESÕES: APLICABILIDADE DA ENFERMAGEM

Francisca das Chagas Rodrigues

Izonilson dos Santos Pimentel

Juscielza Almeida dos Santos

Karla Michele Dourado do Vale

Pedro Henrique Maia Souza

Valéria Arnaud de Melo

Valéria Soares Queiroz

Leandro Silva Pimentel

DOI: 10.47094/978-65-88958-83-4/742-752

CAPÍTULO 61 .753

A IMPORTÂNCIA DA ENFERMAGEM GERONTOLÓGICA NO CUIDADO DO IDOSO DIABÉTICO

Sabrina Thais de Paula Oliveira

Vitória Nicoly Costa de Vasconcelos

Walter Brhemen da Silva Carneiro

Kadmiel Cândido Chagas

DOI: $10.47094 / 978-65-88958-83-4 / 753-766$

\section{SUMÁRIO}




\title{
ASSISTÊNCIA DE ENFERMAGEM AS GESTANTES DIANTE DA PANDEMIA DA
}

COVID-19

\author{
Amanda de Melo Farias ${ }^{1}$ \\ Centro Universitário do Norte (UNINORTE), Manaus, Amazonas. \\ http://orcid.org/0000-0003-0639-3100 \\ Erika Regina Nunes dos Santos ${ }^{2}$ \\ Centro Universitário do Norte (UNINORTE), Manaus, Amazonas. \\ http://orcid.org/0000-0002-4639-4338
}

Geovanne Lima dos Santos ${ }^{3}$

Centro Universitário do Norte (UNINORTE), Manaus, Amazonas.

http://orcid.org/0000-0002-1353-4947

\section{Maycon Henrique Garcia Fonseca ${ }^{4}$}

Centro Universitário do Norte (UNINORTE), Manaus, Amazonas.

http://lates.cnpq.br/6269634871566049

Oliver Khristian Caldas do Nascimento ${ }^{5}$

Centro Universitário do Norte (UNINORTE), Manaus, Amazonas.

http://lates.cnpq.br/7426045999097856

\section{Stefanie Lima Brandão ${ }^{6}$}

Centro Universitário do Norte (UNINORTE), Manaus, Amazonas.

http://orcid.org/0000-0002-0635-7006

Thayanne Rafaela Mota Bandeira ${ }^{7}$

Centro Universitário do Norte (UNINORTE), Manaus, Amazonas.

http://lates.cnpq.br/1241554637597287

\section{Zegilson Ferreira Delmiro ${ }^{8}$}

Centro Universitário do Norte (UNINORTE), Manaus, Amazonas.

http://lates.cnpq.br/6794994960350197

\section{Kadmiel Cândido Chagas ${ }^{9}$}

Centro Universitário do Norte (UNINORTE), Manaus, Amazonas.

http://lates.cnpq.br/0286771587084599

\section{PESQUISA, SAÚDE E GRADUAÇÃO:}


RESUMO: COVID-19 ou o novo coronavírus (SARS-COV2), ganhou espaço em âmbito mundial de forma rápida e letal, sendo que os grupos com comorbidades são mais vulneráveis as formas críticas do vírus, pelo fato do risco elevado de morbimortalidade, as gestantes foram classificadas como grupo de risco para o novo Coronavírus. Este trabalho tem como objetivo enunciar a produção de conhecimento sobre as recomendações para o cuidado à mulher no parto e puerpério e ao recémnascido $(\mathrm{RN})$ frente à nova pandemia do corona vírus, sendo relevante pelo fato de ter o intuito de buscar e construir entendimento sobre a assistência de enfermagem as gestantes nas melhores práticas durante a pandemia da COVID-19. Tratando-se de uma revisão integrativa sobre a assistência de enfermagem as gestantes diante da pandemia da COVID-19, que visa uma síntese do conhecimento sobre o assunto. Utilizaram-se as bases de dados nacionais disponíveis na BVS, com os critérios de inclusão e exclusão entre os anos de 2016 á 2021. Os resultados nos levaram a ressaltar a importância do cuidado especial com as gestantes na pandemia, pois além de encontrarem fragilizadas pelo próprio momento do parto, ainda tem aflições causadas pelo atual cenário.

DESCRITORES: Assistência de Enfermagem. COVID 19. Gestantes.

\section{NURSING ASSISTANCE FOR PREGNANT WOMEN AGAINST THE COVID-19 PANDEMIC}

ABSTRACT: COVID-19 or the new coronavirus (SARS-COV2), quickly and lethally gained worldwide space, and groups with comorbidities are more vulnerable to critical forms of the virus, due to the high risk of morbidity and mortality, pregnant women were classified as a risk group for the new Coronavirus. This paper aims to enunciate the production of knowledge about the recommendations for the care of women in childbirth and puerperium and the newborn (NB) in the face of the new coronavirus pandemic, being relevant because it has the intention to seek and build understanding of nursing care for pregnant women in best practices during the COVID-19 pandemic. This is an integrative review of nursing care for pregnant women in the face of the COVID-19 pandemic, which aims at a synthesis of knowledge on the subject. The national databases available in the VHL were used, with the inclusion and exclusion criteria between the years 2016 to 2021 . The results led us to emphasize the importance of special care for pregnant women in the pandemic, as they are also weakened by the time of childbirth, still has afflictions caused by the current scenario.

DESCRIPTORS: Nursing Assistance. COVID-19. Pregnant women.

\section{INTRODUÇÃO}

O mundo está passando pela mais grave pandemia de uma doença infecciosa causada por um novo coronavírus (SARS-CoV-2). A COVID-19 representa a maior esfinge mundial da saúde publica nos últimos 100 anos sendo potencialmente letal comparado apenas com a gripe espanhola que matou cerca de 25 milhões de pessoas entre 1918 e 1920 (ROTHAN; BYRAREDDY, 2020). 
A infecção pela Síndrome Respiratória Aguda Grave (SARS-CoV-2), é responsável por atentar um quadro inflamatório conhecido como a doença da Coronavírus 2019 (COVID-19), nomeado pela Organização Mundial da Saúde (OMS) (LIU et al., 2020) iniciou na Província de Hubei, na República Popular da China (WU et al., 2020;). Devido à rápida disseminação geográfica da doença, em 11 de março de 2020, a OMS classificou a doença causada pelo Coronavírus 2019 como uma pandemia, quando já havia mais de 118 mil infectados, dos quais 4.291, pessoas vieram a óbito pelo Coronavírus (OMS, 2020).

A alta carga viral no trato respiratório superior é um fator importante na transmissibilidade do COVID-19 mesmo entre pacientes pré-sintomáticos, que o distingue de outras doenças respiratórias. Os diagnósticos baseados em sintomas são mais difíceis, principalmente tratando-se de idosos, pois muitos não apresentam febre, têm tosse crônica por outras patologias ou apresentam dispneia aos esforços por insuficiência cardíaca prévia (THE LANCET, 2020).

A SARS-CoV-2 causa desde sintomas leves como febre, coriza, congestão nasal, dispneia, mal-estar, mialgia e perda do paladar, até sintomas graves como a Síndrome Respiratória Aguda Grave (SARG), sendo que as complicações são mais comuns e até letais em pessoas com morbidades (RODRIGUEZ et al., 2020). O MS inseriu as mulheres durante o ciclo gravídico-puerperal e os Recém-Nascidos (RN) na classificação como grupos de risco, uma vez que o quadro clínico desses indivíduos pode ser insultado na compleição de infecção, devido à baixa tolerância à hipóxia e imunidade, que culminam com piores desfechos, comparados à população em geral (BRASIL, 2020).

Estudos apontam que o número registrado de gestantes e recém-nascidos infectados é muito menor do que a população em geral, contudo, gestantes e puérperas não deixam de ser mais vulneráveis à COVID-19 e, quando enfermam, os presságios podem ser mais graves. No pós-parto, é possível ocorrer a transmissão da mãe para o bebê, e como esse oferece maior imaturidade do sistema imunológico, acredita-se que ele seja mais susceptível à infecção pelo SARS-CoV-2. Assim, sugerese evitar a infecção por meio do contato com a mãe, pessoas adjuntas e profissionais portadores do vírus ou doentes (DONG Y et al., 2020).

Cada vez mais, as instituições de atenção à mulher seja ela maternidade ou unidade básicas de saúde, busca qualificar suas equipes, onde o enfermeiro é protagonista do cuidado, da gestão e organização de fluxos e atendimentos ao cliente. Neste sentido, este trabalho tem o objetivo de enunciar a produção de conhecimento sobre as recomendações para o cuidado à mulher no parto, puerpério e ao recém-nascido $(\mathrm{RN})$ diante da pandemia sendo relevante pelo fato de ter o intuito de buscar e construir entendimento sobre a assistência de enfermagem as gestantes nas melhores práticas durante a pandemia da COVID-19.

\section{METODOLOGIA}

Revisão Integrativa da literatura desenvolvida por meio de seis etapas metodológicas (SOUSA, 2017). A pergunta de pesquisa foi elaborada pela estratégia PICo que preconiza como elementos fundamentais: P - População; I- Interesse; e Co - Contexto. Assim, definiram-se os elementos: P - gestante; I - assistência; e Co - COVID-19. Logo, a questão de pesquisa foi: Como organizar a 
assistência nas maternidades em resposta à pandemia pela COVID-19?

A etapa de identificação de estudos envolveu a busca de evidências científicas em diferentes recursos informacionais, a saber: National Library of Medicine National Institutes of Health (PubMed), Biblioteca Científica Eletrônica Virtual (SCIELO), Medical Literature Analysis and Retrieval System Online (MEDLINE) e Literatura Latino - Americana e do Caribe em Ciências da Saúde (LILACS).

Os critérios de inclusão para a seleção dos estudos foram publicações disponíveis na íntegra (artigos originais, revisões de literatura, estudos de casos), artigos publicados na lingua portuguesa e inglesa, disponiveis no ano de 2016 a 2021. Os critérios de exclusão foram publicações duplicadas (manuscritos em duplicidade foram considerados apenas uma vez). Não houve recorte temporal. A fase de busca e de seleção das produções incluídas na revisão foi realizada por duplas de revisores de forma independente para cada recurso informacional. As dúvidas que surgiram nesse processo foram discutidas e resolvidas em um painel entre todos os revisores até o alcance de um consenso.

Por tratar-se de revisão integrativa, a pesquisa não foi submetida ao Comitê de Ética em Pesquisa, porém foram mantidas as ideias dos autores das publicações utilizadas no desenvolvimento deste estudo. Para estratégias de buscas utilizamos Descritores em Ciências da Saúde (Decs) junto aos conecetores boolenados AND: "Assistencia de Enfermagem" AND "Gestantes" AND "COVID-19" AND "Pandemia". A partir da busca, foram encontradis 542 estudos, nos quais 7 selecionados para este trabalho estando de acordo com o tema proposto conforme fluxograma da figura 1.

Figura 1: Fluxograma do processo de identificação, seleção e inclusão dos estudos. Adaptação da Recomendação PRISMA. Manaus, AM, Brasil, 2021.

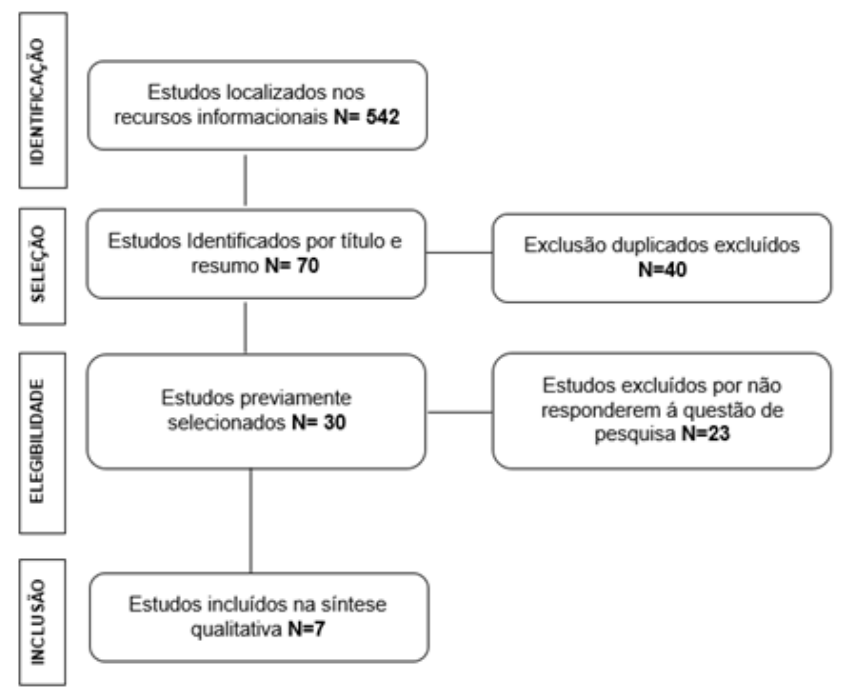




\section{RESULTADOS}

Os diferentes cruzamentos em dupla geraram um total de 542 títulos e resumos lidos, a partir dos quais 70 publicações foram identificadas inicialmente pela aproximação com o foco no estudo. Posteriormente, com a exclusão de 40 publicações duplicadas devido ao critério de exclusão, restaram 30 artigos que foram lidos na íntegra.

Destes 7 estudos compuseram a amostra final, uma vez que 23 foram excluídos por não responderem á questão de pesquisa. Para a seleção dos estudos, utilizaram-se as recomendações Preferred Reporting Items for Systematic Reviews and Meta-Analyses (PRISMA), uma diretriz que tem como objetivo ajudar autores a melhorar a qualidade dos relatos de revisões dessa natureza. $\mathrm{O}$ PRISMA é composto de um checklist de 27 itens e um diagrama de fluxo de seleção das publicações, apresentado na Figura 1.

Todas as publicações foram entre os anos de 2016 á 2021. A maior parte foi produzida por pesquisadores da China 5 (71,42\%), seguidos de pesquisadores brasileiros, 2 (28,57\%).

Quadro 1: Síntese dos artigos da revisão. Manaus, AM, Brasil, 2021.

\begin{tabular}{|c|c|c|c|}
\hline Título & Autores - Ano & Objetivo & Desfecho \\
\hline $\begin{array}{l}\text { O MEDO DO } \\
\text { EM } \text { PARTO } \\
\text { PANDEMIA DO NOVO } \\
\text { CORONAVÍRUS }\end{array}$ & $\begin{array}{ll}\text { SOUTO } & \text { S, } \\
\text { ALBUQUERQUE } & \text { R, } \\
\text { PRATA A, } 2020 & \end{array}$ & $\begin{array}{l}\text { Refletir acerca } \\
\text { da forma como } \\
\text { a pandemia do } \\
\text { novo corona vírus } \\
\text { desencadeou ou } \\
\text { acentuou o medo do } \\
\text { parto nas gestantes e } \\
\text { afetou as práticas de } \\
\text { assistência ao parto. }\end{array}$ & $\begin{array}{l}\text { O período atual acentuou um problema } \\
\text { crónico: um sistema paternalista das } \\
\text { instituições de saúde na abordagem ao } \\
\text { parto, adensado por níveis adicionais } \\
\text { de medo nas gestantes. Nesse contexto, } \\
\text { abordar o medo do parto significa não } \\
\text { abdicar da promoção de experiências } \\
\text { seguras e positivas de parto para as } \\
\text { mulheres. }\end{array}$ \\
\hline $\begin{array}{lr}\text { ASSISTÊNCIA ÁS } & \text { Á } \\
\text { GESTANTES } & \text { E } \\
\text { RECÉM-N A SCIDOS } \\
\text { NO CONTEXTO DA } \\
\text { INFECÇÃO COVID-19: } \\
\text { UMA REVISÃO } \\
\text { SISTEMÁTICA }\end{array}$ & $\begin{array}{l}\text { RONDELLI G, } \\
\text { JARDIM D, HAMAD } \\
\text { G, et al., 2020. }\end{array}$ & $\begin{array}{l}\text { Explorar e sintetizar } \\
\text { as evidências } \\
\text { disponíveis na } \\
\text { literatura científica } \\
\text { voltadas para a } \\
\text { atenção e o manejo } \\
\text { de gestantes e recém- } \\
\text { nascidos suspeitos ou } \\
\text { infectados pelo vírus } \\
\text { SARS-CoV-2. }\end{array}$ & $\begin{array}{l}\text { No Brasil, apesar da infecção } \\
\text { COVID-19 ser de notificação } \\
\text { compulsória, até o momento não } \\
\text { existem detalhamento dos casos em } \\
\text { gestantes e recém-nascidos. Isso } \\
\text { dificulta a criação e a implementação } \\
\text { de ações capazes de interromper o } \\
\text { ciclo de contágio, bem como não } \\
\text { colabora para adequada organização } \\
\text { dos serviços de assistência à saúde, } \\
\text { comprometendo a eficácia do } \\
\text { enfrentamento da pandemia, tanto } \\
\text { pelos órgãos públicos e profissionais da } \\
\text { saúde, quanto pela própria população. }\end{array}$ \\
\hline
\end{tabular}




\begin{tabular}{|c|c|c|c|}
\hline $\begin{array}{l}\text { ESTRATÉGIAS PARA } \\
\text { REORGANIZAÇÃ O } \\
\text { DA ASSITÊNCIA DE } \\
\text { ENFERMAGEM Á } \\
\text { SAPUDE MATERNA } \\
\text { FRENTE Á PANDEMIA } \\
\text { COVID-19 }\end{array}$ & $\begin{array}{l}\text { SANGOI K, SILVA } \\
\text { N, KINLSKI S, et al., } \\
2020 .\end{array}$ & $\begin{array}{l}\text { Realizar uma revisão } \\
\text { narrativa da literatura } \\
\text { sobre a reorganização } \\
\text { e assistência nas } \\
\text { maternidades frente } \\
\text { à pandemia da } \\
\text { Covid-19 }\end{array}$ & $\begin{array}{l}\text { É de extrema importância que as } \\
\text { maternidades se preparem para } \\
\text { uma assistência rápida e eficaz, a } \\
\text { fim de evitar ao máximo uma longa } \\
\text { permanência dos pacientes em } \\
\text { ambiente hospitalar, dando ênfase às } \\
\text { medidas de prevenção do coranavírus. } \\
\text { As recomendações contidas neste } \\
\text { artigo relatam as mudanças de várias } \\
\text { instituições que se empenharam para } \\
\text { colocar em prática descobertas sobre a } \\
\text { repercussão desta doença em gestantes } \\
\text { e puérperas. Assim, a pandemia da } \\
\text { Covid-19 deverá fortalecer o trabalho } \\
\text { nas maternidades entre a equipe de } \\
\text { saúde e os gestores. }\end{array}$ \\
\hline $\begin{array}{l}\text { RECOMENDAÇÕES } \\
\text { DE CUIDADOS PARA } \\
\text { P A R T U R I E N T E S } \\
\text { E PUÉRPERAS E } \\
\text { RECÉM-NASCIDO19: } \\
\text { DURANE A PANDEMIA } \\
\text { COVID-19 UMA } \\
\text { REVISÃO DO ESCOPO }\end{array}$ & $\begin{array}{l}\text { MASCARENHAS, V. } \\
\text { H. A, BECKER A.C; } \\
\text { VENCANCIO K. C. } \\
\text { M, ET AL, } 2020\end{array}$ & $\begin{array}{l}\text { Mapear a produção de } \\
\text { conhecimento sobre } \\
\text { as recomendações } \\
\text { para assistência ao } \\
\text { parto, puerpério } \\
\text { e cuidados com o } \\
\text { recém-nascido em } \\
\text { face da pandemia do } \\
\text { novo coronavírus. }\end{array}$ & $\begin{array}{l}\text { No ciclo gravídico-puerperal deve- } \\
\text { se prevenir a transmissão do vírus, } \\
\text { avaliar a necessidade da interrupção } \\
\text { da gestação, reduzir a circulação } \\
\text { de pessoas, evitar o contato pele a } \\
\text { pele e o parto na água, preferir a } \\
\text { anestesia peridural à anestesia geral, } \\
\text { manter a mulher recém-nascido } \\
\text { do recém-nascido e estimular a } \\
\text { amamentação. Estudos futuros sobre } \\
\text { puxo dirigido, parto instrumental, } \\
\text { clampeamento tardio do cordão } \\
\text { umbilical e banho imediato do recém- } \\
\text { nascido são repassados. }\end{array}$ \\
\hline 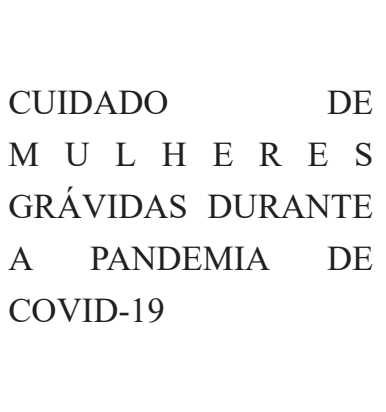 & $\begin{array}{lr}\text { ROCHELSON B, } \\
\text { NIMAROFF } \\
\text { COMBS A, et al, } 2020\end{array}$ & $\begin{array}{l}\text { Facilitar a assistência } \\
\text { á maternidade me } \\
\text { nossos hospitais, } \\
\text { como recursos } \\
\text { significativamente } \\
\text { limitados em pessoal, } \\
\text { equipamento e } \\
\text { espaço. }\end{array}$ & $\begin{array}{l}\text { As lições aprendidas durante a } \\
\text { resposta a esse desafio provavelmente } \\
\text { serão valiosas no período pós- } \\
\text { COVID-19 e devem orientar as } \\
\text { respostas dos serviços obstétricos a } \\
\text { futuras pandemias ou a outras crises } \\
\text { inesperada. }\end{array}$ \\
\hline $\begin{array}{lr}\text { A S S I S T Ê N C I A } \\
\text { O B S T E T R I C A } \\
\text { A } & \text { PORTADRA } \\
\text { DE } & \text { COVID-19: } \\
\text { UMA } & \text { REVISÃO } \\
\text { INTEGRATIVA }\end{array}$ & $\begin{array}{l}\text { FROTA, MARIA } \\
\text { ALBUQUERQUE, et } \\
\text { al., } 2020\end{array}$ & $\begin{array}{l}\text { Busca de estudos } \\
\text { que tratam sobre a } \\
\text { assistência obstétrica } \\
\text { realizada á portadora } \\
\text { de COVID-19. }\end{array}$ & $\begin{array}{l}\text { De acordo com à situação pandêmica } \\
\text { do coronavírus, a assistência obstétrica } \\
\text { encontra-se na incerteza, pois há } \\
\text { divergência no cuidado, relativos } \\
\text { aos protocolos preconizados em } \\
\text { cada instituição, alguns obedecendo } \\
\text { aos critérios específicas próprios do } \\
\text { hospital. }\end{array}$ \\
\hline
\end{tabular}




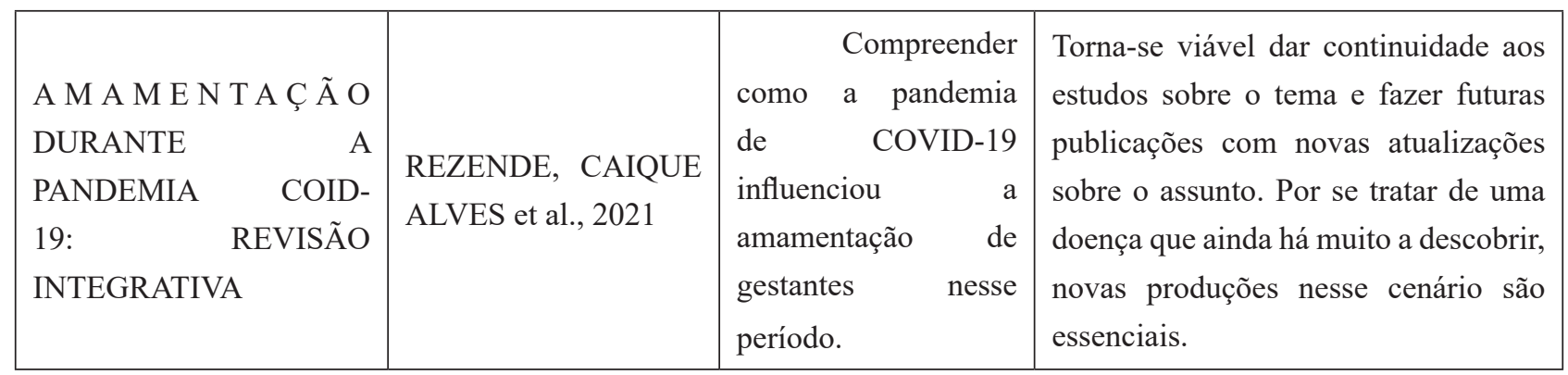

\section{DISCUSSÃO}

Demanda resiliência do profissional enfermeiro responsável por cuidar da mulher gestante em tempos de COVID-19. Durante o pré-natal e o atendimento das gestantes muitos países têm mudado o modelo de manobra para o atendimento das mesmas (ALEX et al., 2020). Com a pandemia da COVID-19 reforçou-se a necessidade do sistema de saúde evoluir, tornando-se mais populares o uso da tecnologia, resultando da utilização da telemedicina (SOUZA et al., 2021).

No entanto, há preocupação quanto a qualidades de assistência prestada, uma vez que muitas das gestantes dependem principalmente dos serviços públicos de saúde. Aos atendimentos presenciais quando realizados, a recomendação é de manter a preocupação de contato com o uso de equipamentos de proteção individual, pelo profissional da saúde, e mascaras cirúrgica pelas gestantes, a porta de entrada para as gestantes nos serviços de saúde vem ser diferenciada dos demais pacientes (BRASIL, 2020).

A assistência obstétrica encontra-se na incerteza devido à situação pandêmica da COVID-19, uma vez que há divergência nos cuidados relativos aos protocolos recomendados em cada instituição, alguns obedecendo aos critérios específicos de protocolos do próprio do hospital (FROTA et al., 2020). Além das dificuldades que podem acontecer durante a gestação, no ciclo gravídico-puerperal deve-se precaver a transmissão do vírus, aferir a necessidade da interrupção da gestação, abater a circulação de pessoas, impedir o contato pele a pele e o parto na água, optar pela anestesia peridural à anestesia geral, e estimular a amamentação (MASCARENHAS et al., 2020).

É de extrema acuidade que as maternidades se preparem para uma assistência rápida e eficaz, a fim de evitar ao máximo uma alongada permanência dos pacientes em ambiente hospitalar, dando ênfase às medidas de prevenção do corona vírus. As recomendações debeladas neste artigo relatam as alterações de várias instituições que se compeliram para colocar em prática descobertas sobre a repercussão desta doença em gestantes e puérperas. Contudo, diante da pandemia o trabalho nas maternidades deveram ser fortalecidas entre as equipes de enfermagem, os demais profissionais e gestores (SANGOI et al., 2020).

As lições aprendidas durante a resposta a esse desafio provavelmente serão valiosas no período pós-COVID-19 e devem orientar as respostas dos serviços obstétricos a futuras pandemias ou a outras crises inesperadas (ROCHELSON et al., 2020). 
No Brasil, apesar da infecção COVID-19 ser de notificação compulsória, até o momento não existem detalhamento dos casos em gestantes e recém-nascidos. Isso bloqueia a criação e a implementação de ações capazes de interromper o ciclo de contaminação, bem como não colabora para adequada organização dos serviços de assistência à saúde, comprometendo a eficácia do enfrentamento da pandemia, tanto pelos órgãos públicos e profissionais da saúde, quanto pela própria população (RONDELLI et al., 2020).

Portanto, todos os artigos ressalvam que o cuidado característico com as gestantes na pandemia é importante, pois além de encontrarem fragilizadas pelo próprio momento do parto, ainda tem aflições originadas pelo atual cenário. O cuidado da assistência de enfermagem e dos demais profissionais de saúde são importantes levando uma maior segurança á mulher. (COSTA et al., 2018).

Torna-se viável dar continuidade aos estudos sobre o tema e fazer futuras publicações com novas atualizações sobre o assunto. Por se tratar de uma doença que ainda há muito a descobrir, novas produções nesse cenário são essenciais (REZENDE et al., 2021).

\section{CONSIDERAÇÕES FINAIS}

A gravidez entrou para o quadro de grupo de risco devido a maior suscetibilidade ao novo corona vírus no período de gravidez ao pós parto e para o recém-nascido., o medo e a angustia dos possíveis riscos de transmissibilidade assolam as gestantes.

A intervenção da enfermagem diminui os impactos da doença em relação mãe-filho, visando estratégias que diminuam o medo e a angustia, gerando um bem estar das gestantes durante esse período. Gestantes representam um grupo de população com particularidades, principalmente ligadas ás suas alterações fisiológicas e imunológicas. Além disso, a necessidade de proteger o feto representa uma maior responsabilidade com relação á prestação de assistência.

\section{DECLARAÇÃO DE INTERESSES}

Nós, autores deste artigo, declaramos que não possuímos conflitos de interesses de ordem financeira, comercial, político, acadêmico e pessoal.

\section{REFERÊNCIAS}

BRASI. Ministério da Saúde. Secretaria de atenção primária pa saúde. Atenção as gestantes no contexto da infecção COVID-19 causada pelo novo coronavírus (Sars-Cov-2). NOTA TÉCNICA No 7/2020- COSMU/CGCIVI/DAPES/SAPS/MS. Brasília, DF, 2020.

ESTRELA, FERNANDA MATHEUS; SILVA, KEILE KEMYLY ASSIS DA; CRUZ, MONIKY ARAÚJO DA; GOMES, NADIRLENE PEREIRA. Gestantes no contexto da pandemia da Covid-19: reflexões e desafios. Physis, Rio de Janeiro, ano 2020, v. 30, n. 2, p. 3, 24 jul 2020

GOIS JT, VIEIRA BC, DIAS FSM, MELO CCA, OLIVEIRA BG, SANTOS APB. Gestantes 
COVID19 positivo, trabalho de parto e risco de transmissão vertical: revisão sistemática. SaudColetiv (Barueri) [Internet]. 2021

LIU X, CHEN M, WANG Y, SUN L, ZHANG J, SHI Y, et al. Prenatal anxiety and obstetric decisions among pregnant women in Wuhan and Chongqing during the COVID-19 outbreak: a cross-sectional study. BJOG [Internet]. 2020

MASCARENHAS VHA, CAROCI-BECKER A, VENÂNCIO KCMP, BARALDI NG, DURKIN AC, RIESCO MLG. COVID-19 and the production of knowledge regarding recommendations during pregnancy: a scoping review. Rev. Lat. Am Enfermagem [Internet]. 2020

NAKAGAWA K, UMAZUME T, MAYAMA M, CHIBA K, SAITO Y, KAWAGUCHI S, et al. Feasibility and safety of urgently initiated maternal telemedicine in response to the spread of COVID-19: A 1-month report. J Obstet Gynaecol Res. [Internet]. 2020

ORGANIZAÇÃO MUNDIAL DA SAÚDE. Ações essenciais de nutrição: melhorar a saúde e nutrição materna, neonatal, infantil e infantil. [Internet]. Genebra: OMS; 2013 [citado em 7 de abril de 2020]. Disponível em: https://apps.who.int/iris/bitstream/handle/10665/84409/9789241505550 eng.pdf $[\underline{\text { Links }}$

REZENDE, CA.; BARBOSA, TCP.; BERNARDES, IA de S..; SANTOS, IT dos.; LIMA, MRM.; CAMILO, AAV.; BERNARDO, GJR.; OLIVEIRA, MM. Amamentação durante a pandemia de COVID-19: revisão integrativa. Pesquisa, Sociedade e Desenvolvimento , [S. l.] , v. 10, n. 4, pág. e46310414475, 2021. DOI: 10.33448 / rsd-v10i4.14475. Disponível em: https://www.rsdjournal. org/index.php/rsd/article/view/14475. Acesso em: 26 set. 2021.

RODRIGUEZ-MORALES AJ, CARDONA-OSPINA JA, GUTIÉRREZ-OCAMPO E, HOLGUINRIVERA Y, ESCALERA-ANTEZANA JP, ALVARADO-ARNEZ LE, et al. Características clínicas, laboratoriais e de imagem do COVID-19: uma revisão sistemática e meta-análise. Travel Med Infect Dis. 2020; 34: e101623. doi: 10.1016 / j.tmaid.2020.101623.

ROTHAN HA, BYRAREDDY SN. The epidemiology and pathogenesis of coronavirus disease (COVID-19) outbreak. J Autoimmun. 2020;109:102433.

THE LANCET. COVID-19: protecting health-care workers. The Lancet. 2020;395(10228):922.

SOUZA SS, CUNHA AC, SUPLICI SER, ZAMPROGNA KM, LAURINDO DLP. Influência da cobertura da atenção básica no enfrentamento da COVID-19. J Health NPEPS [Internet]. 2021

WU JT, LEUNG K, LEUNG GM. Nowcasting and forecasting the potential domestic and international spread of the 2019-nCoV outbreak originating in Wuhan, China: a modelling study. The Lancet [Internet]. 2020 [cited 2020 Mar 24]; 395(10225):689-697. DOI: https://doi.org/10.1016/S0140$\underline{6736(20) 30260-9 .}$. 


\section{Índice Remissivo}

A

Acidente Vascular Cerebral 624, 625, 626, 627, 628, 630, 631, 632

Acompanhamento da gestante 317, 319, 469, 515

Acompanhamento paterno no período inicial da gestação 317, 318, 320

Adoecimento físico e psicológico 369, 378

Afastamento do trabalho 519, 525

Agentes biológicos 442, 452

AIDS 150, 152, 154, 256, 257, 258, 259, 262, 263, 264, 266, 267, 268, 291, 293

Amamentação 179, 217, 218, 219, 221, 222, 223, 224, 225, 226, 310, 312, 325, 389, 459, 460, 491

Amamentação exclusiva 217, 218, 219, 221, 222, 223, 224, 226

Ambiente hospitalar 81, 163, 166, 169, 188, 192, 210, 216, 242, 314, 413, 417, 419, 421, 459, 460, 473, 506, 537, 571, $636,666,672,689$

Anorexia 353, 354, 361, 365

Antivacina 295

Aperfeiçoamento profissional 52, 233

Aplicativos de relacionamentos 282, 285

Apoio à mulher durante a gravidez 317,325

Assistência ao estomizado 657

Assistência ao paciente 62, 64, 69, 79, 155, 234, 241, 275, 521, 595, 657, 712

Assistência de enfermagem 487, 525, 656, 659

Assistência de qualidade no pré-natal e parto 692, 697, 700

Assistência do pré-natal 155, 158, 174

Assistência humanizada 118, 173, 174, 176, 181, 182, 184, 188, 189, 190, 191, 192, 193, 262, 266, 312, 388, 471, 485, $517,546,699,701,726,728$

Assistência humanizada à parturiente 183

Atenção a saúde 74, 81, 109

Atenção Primária a Saúde 40, 42, 43, 132

Atendimento obstétrico 173

Atuação do enfermeiro 51, 53, 54, 59, 82, 105, 121, 127, 138, 141, 182, 184, 185, 188, 193, 261, 262, 264, 266, 267, $268,271,274,276,281,335,521,524,531,581,584,586,588,657,698,702,743,744,759$

Autismo 83, 84, 85, 87, 88, 89, 90, 93, 94

Autocuidado 6, 36, 126, 135, 138, 139, 140, 199, 202, 247, 266, 331, 370, 558, 559, 581, 647, 648, 657, 661, 662, 664, $666,667,668,710,712,713,758,759,763,765,766$

Avanço da pandemia 368, 371, 378

AVC e a Covid-19 624

$\mathrm{B}$

Bactericida 743, 747, 749

Bem-estar biopsicossocial 562, 570

Benefícios da musicoterapia 494

Bulimia 353, 354, 361, 365

Bullyng 353,354 
Câncer 53, 56, 57, 60, 61, 245, 269, 270, 272, 274, 281, 574, 577, 582, 671, 675

Câncer de colo do útero 51, 52, 53, 54, 59, 575, 576, 579, 580, 582, 583

Câncer de mama 218, 222, 245, 247, 250, 251, 252, 254, 255, 575

Câncer de pele 269, 270, 271, 272, 274, 278, 281

Câncer uterino 574

Capacitação 340, 600

Capacitação em saúde 340

Categorias profissionais $30,173,334$

Cicatrizações 743,745

Ciclo gradívico-puerperal 307

Ciências da saúde 31, 38, 43, 51, 54, 64, 76, 97, 98, 108, 119, 133, 147, 157, 219, 230, 239, 247, 248, 267, 285, 286, $294,297,308,342,343,356,357,366,384,394,396,397,422,425,438,442,445,446,457,481,483,484$, $497,498,512,519,521,535,552,562,563,564,584,586,598,603,615,656,659,670,673,684,695,706$, $726,729,765$

Classificação de risco 370, 584, 586, 588, 589, 590, 591, 592, 593, 594, 595, 596, 597, 598

Cobertura vacinal $296,299,302,303,443,447,448,450,451,715,717,723$

Comorbidades $125,197,202,278,455,524,526,559,630,631,659,705$

Compartilhamentos 95, 97, 395

Complicações 203, 206, 208, 210, 213, 216, 524, 763

Comportamento 34, 38, 84, 89, 91, 139, 141, 145, 146, 149, 151, 152, 179, 199, 289, 292, 324, 328, 353, 358, 359, $361,362,363,364,365,394,396,404,504,528,619,620,672,737$

Comportamento sedentário 354, 359, 363, 364

Comportamento sexual 146, 149, 353, 358, 359, 364

Comportamento violento $353,362,619$

Condutas do enfermeiro $612,614,615$

Condutas em primeiros socorros $340,342,351$

Conhecimento em primeiros socorros 339, 342, 345

Conhecimento em primeiros socorros de professores e colaboradores 340, 347

Conhecimento prévio da episiotomia 422

Construção da tecnologia educativa em saúde 340, 347, 349

Consumo de bebidas alcoólicas 353

Contaminação $74,75,76,102,258,279,287,290,291,377,461,473,519,525,528,529,569,639,642,652,653$

Coronavírus (sars-cov2) 455

Covid-19 31, 33, 34, 38, 39, 78, 79, 82, 95, 96, 97, 99, 100, 102, 103, 104, 372, 373, 377, 379, 399, 401, 404, 405, 406, $443,444,459,461,519,520,521,524,527,528,529,531,565,567,568,604,605,606,607,610,624,625$, $626,627,628,629,630,631,632,633,635,636,637,640,641,642,643,645,647,648,650,651,652,653$, $654,655,715,716,717,719,720,721,722,723,724$

Covid-19 e o Acidente Vascular Cerebral (AVC) 624, 627

Criança autista $83,90,91,92$

Cuidado pré-natal 155,157

Cuidados da criança 83,91

Cuidados em UTIN 681, 683

Cuidados paliativos 237, 239, 241, 242, 243, 244, 245, 247, 248, 250, 252, 253, 254, 255, 274, 670, 671, 672, 674, 675, 676 
Cuidados sobre o diabetes 704

$\mathrm{D}$

Departamento de Análise e Tabulação de Dados do Sistema Único de Saúde (DATASUS) 162

Dermatologia 275, 276, 281, 330, 333, 334

Dermatologia em enfermagem 269

Desafios do enfermeiro intensivista 519, 521

Desigualdade social 170, 369, 374, 376, 378

Desinformação antivacina na contemporaneidade 715

Despreparo dos profissionais da saúde 369, 378

Diabetes 117, 123, 125, 127, 174, 194, 195, 196, 197, 198, 199, 200, 201, 202, 203, 204, 205, 222, 276, 558, 594, 625, $630,704,705,706,708,709,710,711,712,713,714,747,751,754,755,756,758,759,760,761,764,765$, 766

Diabetes mellitus ii (dm) 194, 195, 196

Diabtes mellitus 704, 705

Diagnóstico 41, 46, 51, 53, 54, 56, 58, 59, 69, 83, 84, 85, 86, 87, 88, 89, 90, 91, 92, 93, 94, 107, 111, 113, 122, 124, $146,150,151,153,159,160,200,203,204,241,243,253,263,264,265,267,269,270,278,279,284,300$, $511,575,576,577,631,652,658,666,671,675,706,737,747$

Dificuldades no ato sexual 145, 149

Distanciamento social 33, 96, 103, 369, 375, 376, 377, 378, 379, 605, 720, 722

Distúrbio 37, 83, 84, 89, 92, 196, 212, 755, 761

Doenças crônicas 131, 194, 196, 353, 355, 358, 359, 360, 365, 367, 413, 417, 505, 558, 628, 754, 761

Doenças infecciosas 117, 123, 127, 194, 196, 296, 355, 443, 448, 449, 450, 451

Doenças vasculogênicas 763

Doença terminal 238, 239

Drogas 35, 37, 151, 178, 354, 355, 357, 361, 362, 364, 499, 515

E

Eclampsia 123, 125, 163, 168, 212, 585, 594, 597

Educação em saúde 52, 59, 106, 113, 126, 139, 141, 173, 177, 201, 202, 211, 215, 223, 253, 261, 265, 266, 267, 292, $303,305,345,351,359,370,374,380,400,403,436,451,467,468,469,556,559,578,579,666,708,711$, $715,717,723,759$

Efeitos psicológicos 29

Enfermagem 6, 28, 29, 30, 31, 33, 34, 35, 36, 37, 38, 40, 41, 42, 45, 46, 47, 48, 49, 50, 53, 55, 56, 57, 59, 61, 63, 66, $68,70,76,78,79,80,81,82,83,92,93,104,105,107,108,110,112,113,117,121,123,124,126,127,135$, $136,138,140,141,142,143,149,151,153,172,175,176,177,179,180,183,184,185,188,189,190,191$, $192,193,198,200,215,224,227,228,229,230,231,232,233,234,235,236,241,242,243,245,246,248$, $250,251,252,253,254,255,258,261,263,264,265,266,267,268,269,271,272,273,274,275,276,277$, $278,279,280,281,286,288,293,306,308,310,312,313,315,316,319,322,324,327,328,329,330,331$, $332,333,335,336,337,338,343,347,352,357,384,386,388,390,391,392,398,407,408,409,410,412$ 413, 414, 415, 416, 417, 418, 419, 420, 421, 426, 429, 431, 435, 438, 439, 447, 452, 455, 456, 460, 461, 463, 465, 466, 467, 469, 470, 471, 472, 474, 475, 476, 477, 478, 479, 481, 482, 483, 484, 485, 486, 487, 488, 490, $491,506,510,511,515,516,523,524,525,526,527,528,529,530,531,533,534,535,538,540,542,543$, $544,545,546,547,548,549,553,559,562,564,565,566,567,568,569,570,571,577,588,589,590,591$, $592,593,595,596,597,600,601,602,604,605,606,607,608,609,610,612,617,618,619,620,621,622$, $623,626,627,633,635,636,639,640,642,643,647,648,649,650,651,652,653,654,657,659,660,661$,

PESQUISA, SAÚDE E GRADUAÇÃO:

MONOGRAFIAS QUE ENTRELAÇAM E CONTRIBUEM PARA O SER-PROFISSIONAL 
$662,663,664,666,667,668,670,672,673,674,675,676,681,682,683,685,686,687,688,689,690,694$, 697, 698, 700, 702, 703, 704, 705, 706, 707, 708, 709, 710, 711, 712, 713, 714, 717, 718, 719, 721, 722, 724, $726,728,729,730,731,737,738,739,740,741,745,747,749,750,751,753,755,756,757,759,760,761$, $763,764,765,766$

Enfermagem dermatológica 269, 272, 276, 334, 335

Enfermagem estética 330, 333, 336

Enfermeiro na área dermatológica e estética 330,332

Enfermeiro na assistência obstétrica 173

Enfermeiro na unidade de terapia intensiva neonatal 681,683

Enfermeiro no combate à violência obstétrica 692

Enfermeiros intensivistas 519, 528, 529, 530

Enfermeiros no processo de imunização da covid 715,717

Enfrentamento da pandemia 95, 97, 103, 377, 458, 461, 525, 531, 566, 567, 568, 571, 608, 645, 648, 655, 717

Entidades organizacionais de saúde 715,723

Envelhecimento populacional 194, 196, 232

Episiotomia 190, 422, 423, 424, 425, 427, 428, 429, 430, 431, 432, 433, 435, 436, 437, 438, 439, 440, 697, 700

Equipe de enfermagem $29,47,48,121,564,570,685,704,738$

Equipe de saúde $68,173,174,176,179,197,208,214,278,314,459,464,511,516,529,538,540,546,547,548$, $550,554,559,585,620,681,683,689,693,699,704,708,728,749$

Equipe e paciente 173

Equipe multiprofissional 35, 71, 85, 174, 211, 239, 244, 266, 270, 491, 539, 541, 544, 635, 636, 642, 658, 659, 662, $674,682,706,737,760$

Equipe, paciente e familiares 306

Estado pró-inflamatório 625,630

Estado pró-trombótico 625,630

Estágio terminal da doença 237

Estilo de vida 36, 196, 197, 198, 200, 201, 325, 355, 359, 363, 557, 558, 559, 665, 706, 709, 710, 753, 761, 762, 763, 764

Estilo de vida 195

Estilo de Vida Indígena 550, 552

Estomias 657

Estratégia 46, 132, 152, 200, 218, 258, 265, 275, 280, 468, 475, 497, 514, 582, 600, 612, 613, 614, 615, 618, 619, 620, $621,622,661,665,765,766$

Estratégias de capacitação 251, 600

Estresse 29, 33, 34, 36, 38, 70, 71, 80, 88, 91, 100, 102, 178, 223, 313, 370, 390, 407, 408, 409, 410, 413, 414, 415, $416,417,418,419,420,421,490,494,500,503,505,519,528,529,558,570,601,651,652,653,654,743$, 751

Estresse ocupacional 407, 408, 409, 415, 416, 417, 418, 421

Etilismo 550,558

Evolução das fake news 394, 396, 404

Exame papanicolau $51,53,54,56,57,59,61,577,578,579,581,582$

Exame preventivo de câncer de colo uterino (pccu) 574, 580

Exaustão física e mental 37, 520, 530, 653

Excesso de carga horária de trabalho 562, 570

Excesso de peso 550, 558

Expectativa de vida do brasileiro 194, 196 
Fake news 95, 96, 97, 98, 99, 100, 101, 102, 103, 104, 299, 300, 304, 305, 394, 395, 397, 399, 400, 401, 403, 405

Fakes news sobre vacina 295

Falta de conhecimento e informação $354,361,364$

Falta de recursos $48,519,564,570,701$

Fases que antecedem a morte 237, 241

Fatores de risco cardiovasculares 550, 552, 554, 558, 559

Fatores de risco vascular tradicionais 625,630

Fluxos na rotina 519

Fungicida 743, 747, 749

G

Gestação 117, 119, 127, 180, 388

Gestantes diante da pandemia da covid-19 455

Gonorreia 105, 106, 107, 108, 110, 112, 113

$\mathrm{H}$

Hábitos alimentares 126, 195, 197, 201, 202, 353, 358, 361, 363, 706, 754, 762

Hemorragia pós-parto 126, 163

Hesitação vacinal 442, 445, 447

Hipertensão 117, 125, 127, 174, 184, 196, 353, 358, 363, 364, 366, 413, 418, 496, 526, 551, 552, 554, 555, 556, 557, $558,559,560,561,585,594,625,630,708,765$

Hipertensão Arterial Sistêmica 550, 552, 554, 560

HIV 10, 114, 123, 125, 127, 144, 145, 146, 147, 149, 150, 151, 152, 153, 154, 212, 256, 257, 258, 261, 262, 263, 264, 265, 266, 267, 268, 284, 287, 288, 289, 290, 291, 292, 293

Humanização do parto 188, 189, 191, 192, 311, 312, 314, 316, 383, 387, 391, 392, 430, 463, 467, 474, 475, 485, 488, 490

Humanização hospitalar 600

I

Idoso $130,138,143,145,147,227,228,229,230,235,763,765$

Idoso diabético $753,755,756,761,764$

Idosos convivendo com o hiv 145, 146

Idoso soropositivo 256, 258, 259, 263, 265

Impacto na saúde 33, 38, 195

Impactos das fakes news 95

Implantação 40, 41, 42, 43, 46, 47, 48, 49, 56, 307, 340, 351, 390, 448, 453, 488, 514, 537, 547, 589, 592, 593, 597, $606,609,685,721,722,739$

Importância da imunização 442, 451

Importância da presença do pai 317, 319, 325

Imunização 295, 442, 443, 445, 448, 449, 453

Imunização da população 294, 296

Imunobiológicos 74, 75, 296, 299, 447, 450, 723

Imunologia 407, 414

Imunossupressor 407, 409, 410 
Inatividade física $358,363,550,558,762$

Índice de morbidade 105,106

Indígenas com has 550,552

Infecção puerperal $163,168,169$

Infecções sexualmente transmissíveis 106, 107, 111, 113, 114, 150, 153, 156, 160, 256, 262, 266, 268, 282, 283, 284, $289,293,360,575$

Influência da doula $381,383,384$

Infodemia 95, 96, 399, 406

Infraestrutura inadequada 519

Início tardio do pré-natal 510,512

Interação social $83,84,88,89,92,579,728$

Intervenções $34,39,79,82,142,288,336,345,351,590,600,650,659,676,708,763$

Intervenções humanizadoras 600

$\mathrm{L}$

LGBTQIA+ 282, 283, 284, 285, 290, 291, 292

Libido sexual 145

Limitações 83, 92, 109, 152, 170, 229, 238, 275, 373, 601, 639, 644, 666, 674, 711, 761

Linha de frente $28,31,34,80,180,370,375,377,521,527,530,563,564,566,568,569,607,635,647,648,650$, $651,652,653,717$

$\mathrm{M}$

Mãe lactante 217, 219

Medicamentos 74, 76, 92, 146, 151, 152, 178, 190, 196, 200, 214, 228, 238, 275, 324, 377, 472, 475, 500, 505, 511, 706,763

Medicina 66, 67, 69, 73, 105, 110, 330, 331, 336, 337, 357, 389, 395, 402, 412, 429, 438, 467, 472, 474, 475, 481, 484, $494,496,508,523,553,558,577,660,672,703,722,757$

Melanoma 269, 270, 274, 279, 281, 575

Métodos de acolhimento 600

Métodos de humanização 600,608

Ministério da saúde 47, 53, 54, 58, 60, 89, 96, 97, 100, 103, 107, 114, 127, 132, 142, 153, 160, 161, 162, 164, 170, 171, $175,177,180,181,184,192,203,225,228,230,235,244,254,281,300,307,308,309,313,314,315,316$, $328,350,365,369,372,373,376,378,379,382,403,444,447,448,452,461,464,473,477,482,496,507$, $515,516,522,548,575,581,587,590,598,610,622,637,643,648,649,652,654,701,702,718,739,743$, 750,765

Morbidade 111, 124, 125, 194, 196, 207, 208, 279, 313, 323, 443, 555, 711, 754

Morbimortalidade 201, 315, 455, 511, 517, 550, 554, 558, 559, 613, 658

Mortalidade 30, 116, 117, 118, 119, 121, 122, 123, 125, 126, 127, 128, 162, 163, 164, 165, 166, 167, 169, 170, 174, $183,184,194,196,199,207,208,216,222,223,229,258,279,281,307,308,312,313,316,323,376,377$, $382,443,510,511,514,516,517,524,527,530,555,557,560,579,585,586,591,594,629,630,683,711$, $716,722,754,755$

Mortalidade materna 116, 117, 118, 119, 121, 122, 123, 125, 126, 127, 162, 163, 164, 165, 166, 167, 169, 170, 174, 183, 184, 207, 308, 313, 316, 510, 511, 516, 517, 585, 591, 594

Mortalidade materna e neonatal 116, 117, 118, 126, 308, 510, 516, 517

Movimento antivacina 294, 296, 297, 299, 302, 303, 304, 723

Mudanças epidemiológicas no brasil 194, 196

\section{PESQUISA, SAÚDE E GRADUAÇÃO:}


Mulher no pré-natal 117

Múltiplos parceiros 354, 361, 364, 574

Musicoterapia 489, 491, 494, 496, 497, 499, 500, 501, 502, 504, 505, 506, 509

$\mathrm{N}$

Negativamente a opinião pública sobre ciência 394

Neisseria gonorrhoeae 105, 106, 107

Neoplasia mamaria 245, 247, 248

Neurodesenvolvimento 83, 84, 88, 92, 222

Notícias falsas 95, 100, 101, 102, 295, 296, 299, 300, 302, 303, 394, 395, 396, 397, 399, 401, 402, 404

Nutrição do lactente 217

$\mathrm{O}$

Obesidade/sobrepeso 353

Óbito materno e neonatal 116, 118, 121, 124, 126

Óbitos 53, 74, 75, 76, 80, 117, 118, 121, 122, 123, 125, 127, 128, 163, 164, 165, 166, 167, 168, 169, 170, 171, 184, $201,341,348,362,375,511,549,591,755$

Obstetrícia 307, 316, 422, 425, 426, 584, 587, 595, 596

Operacionalização 40, 41, 42, 43, 46, 47, 48, 49, 275, 447, 452, 541, 588, 632

Orientações pré e pós-cirurgia oncótica 269, 280

Ostomizados 657, 659, 662, 665, 668

Ozonioterapia 743, 744, 745, 747, 748

$\mathrm{P}$

Paciente com surdez 726, 728

Paciente oncológico 241, 244, 250, 252, 281, 663, 667, 670, 672, 675

Pacientes adultos ostomizados 656, 659

Pacientes idosos 71, 138, 227, 229, 233, 234, 262, 504, 758, 759, 766

Pacientes terminais 237, 238, 239, 242, 243, 247

Padrão alimentar inadequado 550, 558

Pandemia de covid-19 28, 31, 36, 79, 95, 372, 374, 375, 376, 379, 380, 401, 405

Parto cesáreo 206, 207, 208, 210, 211, 212, 215, 216

Parto humanizado 173, 174, 178, 183, 184, 185, 188, 189, 191, 192, 306, 308, 310, 311, 312, 313, 314, 315, 381, 384, $387,390,436,464,465,469,474,476,481,482,483,485,486,487,489,490,491,698,699,702$

Parto natural 126, 178, 188, 206, 208, 381, 383, 384, 388, 422, 424, 435, 489, 491

Partos prematuros 510, 514, 516

Parturientes 177, 178, 180, 190, 211, 215, 306, 307, 315, 316, 383, 387, 389, 423, 427, 430, 434, 435, 437, 438, 439, $463,467,468,470,472,478,479,482,490,491,592$

Paternidade 318, 320, 327

Patologia 28, 71, 201, 238, 242, 246, 247, 444, 519, 530, 574, 581, 626, 704, 705, 706, 711, 716, 755, 756, 758

Patologias 29, 31, 36, 117, 139, 156, 163, 201, 206, 233, 246, 247, 265, 336, 407, 409, 413, 415, 417, 418, 456, 529, $551,575,625,630,631,666$

Perdas dos pacientes 28

Perfil epidemiológico 108, 123, 131, 159, 162, 164, 170

Período gravídico-puerperal 122, 126, 164, 169, 172, 174, 176, 179

Período pandêmico 369, 648

PESQUISA, SAÚDE E GRADUAÇÃO:

MONOGRAFIAS QUE ENTRELAÇAM E CONTRIBUEM PARA O SER-PROFISSIONAL 
Política nacional de atenção integral à saúde do homem (pnaish) 317, 318, 319

Prática de atividade física 138, 200, 354, 357, 363, 364, 365

Práticas alimentarem 353

Práticas de saúde 42, 130, 139, 387, 392, 549

Práticas éticas 532, 548

Pré e pós-operatório da ostomia 657, 662

Pré-natal tardio 510

Presença do pai no pré-natal 317

Preservativos 107, 145, 146, 149, 152, 291

Prevenção 51, 53, 54, 56, 57, 59, 60, 61, 68, 74, 75, 79, 80, 97, 101, 102, 103, 105, 112, 113, 118, 119, 122, 126, 132, $136,138,139,141,150,153,155,157,160,197,199,201,204,206,208,228,233,247,256,258,259,262$, $265,266,268,271,276,277,278,282,288,289,290,291,293,300,316,319,335,344,348,352,355,358$, $370,371,377,395,396,403,404,434,435,444,447,448,459,460,463,465,468,469,470,471,472,474$, $476,477,478,479,510,511,512,517,551,556,558,559,566,573,575,576,577,578,579,580,581,582$, $583,591,601,606,609,619,620,621,623,641,642,647,652,658,670,672,687,694,702,704,706,708$, $709,710,711,712,713,751,758,759,760,763,764$

Principais riscos existentes no ambiente escolar 340, 347, 348

Procedimento de episiotomia 422

Processo de enfermagem 40, 41, 42, 43, 46, 48, 49, 62, 178, 687

Processo saúde-doença 295, 302, 447, 556, 614, 641, 676, 701, 711

Profissionais de enfermagem 29, 30, 41, 47, 48, 105, 227, 562, 648, 717

Profissionais qualificados em libras 726,739

Programa de Pré-natal masculino 317, 318, 319

Promoção da saúde 130, 133, 142

Protocolos 47, 48, 58, 71, 253, 271, 274, 376, 459, 460, 519, 524, 525, 527, 567, 612, 615, 618, 619, 643, 748

Psicologia 66, 105, 110, 174, 220, 329, 357, 467, 481, 484, 496, 577, 604, 610, 660, 707, 757

Q

Qualidade da coleta 51, 53, 54, 58, 59

Qualidade da humanização 183, 192

Qualidade de vida 36, 80, 81, 95, 97, 113, 118, 131, 132, 140, 141, 149, 151, 169, 194, 196, 197, 198, 199, 200, 201, $202,204,205,229,232,234,238,240,242,243,244,245,247,250,251,252,265,266,269,271,275,277$, $278,355,390,414,415,417,419,465,494,506,514,559,562,567,570,613,626,658,663,664,667,672$, $676,683,700,704,708,711,713,723,754,755,756,758,760,761,763,766$

Qualidade do atendimento 36, 173, 592, 620

$\mathrm{R}$

Recém-nascido 118, 122, 125, 126, 173, 178, 179, 183, 184, 208, 216, 218, 307, 312, 313, 314, 455, 456, 459, 461, $481,489,491,511,682,683,686,687,688,689,690$

Recém-nascidos prematuros 681, 683

Recuperação de pacientes 496, 600, 601, 602, 609

Redes midiáticas 394, 395, 396, 397, 399, 404

Redes sociais 95, 97, 100, 101, 102, 263, 288, 299, 301, 395, 396, 399, 400, 401, 403, 404, 569, 621, 723

Reféns da pandemia 74, 81

Relação pais-filho 317, 325

Relacionamentos geossocial 282

PESQUISA, SAÚdE E GRADUAÇÃO:

MONOGRAFIAS QUE ENTRELAÇAM E CONTRIBUEM PARA O SER-PROFISSIONAL 
Remoção aérea de pacientes em estado crítico 635, 636

Repercussões físicas e emocionais 74, 76

Respeito a vida humana 532

Riscos à gestante 116,118

Riscos em adolescentes 354

S

Saúde da família 510, 622

saúde da gestante e do bebê 510

Saúde da Mulher 52, 54, 127, 180, 307, 323, 481, 514, 517, 518, 574

Saúde da pessoa idosa 130, 132, 133, 141, 142, 232, 235

Saúde da população 132, 150, 152, 164, 294, 296, 297, 303, 404, 704, 706

Saúde de adolescentes escolares 353, 355, 357, 361, 364, 365

Saúde do adolescente 354

Saúde emocional dos profissionais 647,648

Saúde e segurança de profissionais 34, 39

Saúde materno-infantil 311, 381, 390

Saúde mental 33, 34, 35, 36, 37, 38, 74, 76, 78, 79, 80, 81, 82, 95, 99, 101, 102, 317, 321, 325, 355, 359, 363, 375, 377, $380,506,528,571,604,607,647,648,650,651,652,653,654,698,700$

Saúde mental de enfermeiros 74,76

Saúde no cuidado aos indígenas 550, 559

Saúde pública 75, 105, 106, 107, 111, 113, 117, 127, 156, 161, 164, 194, 196, 202, 203, 204, 223, 229, 291, 296, 297, $307,325,330,337,352,368,369,370,371,373,378,379,380,394,396,399,400,403,404,442,443,444$, $445,448,449,475,478,511,530,585,620,635,642,647,653,667,671,693,699,718,722,755,765$

Serviços de transporte aeromédico 635,636

Sexualidade 113, 136, 138, 140, 143, 145, 152, 257, 258, 262, 264, 265, 266, 268, 289, 353, 359, 362, 367, 432, 575

Sexualidade dos idosos 145, 152

Sexualidade precoce 354,359

Sífilis congênita $155,157,160$

Síndrome da Imunodeficiência Adquirida 145, 256, 268, 291

Sistema de Informação Sobre Mortalidade (SIM) 162

Sistema de Informação Sobre Nascidos Vivos (SINASC) 162

Sistema imunológico 31, 107, 112, 407, 409, 418, 444, 456, 574

Situação de violência $612,615,621$

Situações de medo e incertezas 520, 530

Situações de violência contra a mulher $612,614,615,618,620,621$

Suporte Básico de Vida 339, 340, 341, 342, 351

Surdez 727, 740

$\mathrm{T}$

Tabagismo 196, 197, 362, 363, 550, 552, 558, 625, 630, 705

Tabu em relação à vida sexual 256

Tecnologia educativa 339, 342, 347, 348, 349

Terapia ocupacional 66, 105, 110, 357, 481, 484, 660

Terapias complementares 494, 497

Teste de papanicolau 52, 54, 57, 61 
Tipo de parto 178, 206, 211, 215, 216

Trabalho de parto $182,184,185,188,189,191,210,212,213,214,216,313,314,315,321,386,388,389,390,391$, 424, 436, 462, 465, 468, 469, 472, 485, 486, 487, 488, 489, 490, 491, 510, 589, 592, 693, 698, 699

Trabalho em meio à pressão 519

Transmissão do vírus $145,146,459,460,520,563,716$

Transmissão vertical de doença infecciosas 155, 157

Transporte Aeromédico 635, 637

Transtorno do Espectro Autista (TEA) 83, 84, 88, 92

$\mathrm{U}$

Ultrassonografia 62, 63, 64, 67, 68, 69, 72, 73, 323

Unidade de terapia intensiva neonatal 314, 681, 683, 691

Unidades de Terapia Intensiva (UTIs) 519

Unidades obstétricas 584, 586

Uso da ozonioterapia 743, 744, 746, 747, 751

Uso de álcool 178, 354, 361, 364, 711

V

Vacinação da população brasileira 442, 445

Vínculo pai e bebê/ filho 317

Violência 136, 138, 139, 140, 177, 290, 341, 353, 359, 361, 362, 364, 370, 392, 422, 423, 426, 427, 428, 431, 434, 435, 436, 438, 439, 463, 464, 465, 467, 468, 469, 470, 471, 472, 473, 474, 475, 476, 477, 478, 479, 612, 613, 614, $615,617,618,619,620,621,622,623,692,693,694,697,698,699,700,701,702,703$

Violência contra a mulher 612, 613, 614, 615, 617, 618, 619, 620, 621, 623, 693, 698, 703

Violência doméstica $612,618,619,621,622,623$

Violência obstétrica(vo) 422

Viricida 743,749

Vírus da Imunodeficiência Humana 145, 256, 258, 291

Vulnerabilidade $74,76,81,111,118,141,150,151,152,153,159,169,250,252,255,258,267,292,359,361,362$, $370,592,615,676,682,711,761$ 


\section{EDITORA OMNIS SCIENTIA}

editoraomnisscientia@gmail.com Y https://editoraomnisscientia.com.br/

@editora_omnis_scientia @ https://www.facebook.com/omnis.scientia.9 f +55 (87) 9656-3565 @ 


\section{EDITORA OMNIS SCIENTIA}

editoraomnisscientia@gmail.com M https://editoraomnisscientia.com.br/ (4)

@editora_omnis_scientia (0) https://www.facebook.com/omnis.scientia.9 $\mathbf{f}$ +55 (87) 9656-3565@ 